\title{
Optimal Control of a Class of Hybrid Systems
}

\author{
Christos G. Cassandras, Fellow, IEEE, David L. Pepyne, Member, IEEE, and Yorai Wardi
}

\begin{abstract}
We present a modeling framework for hybrid systems intended to capture the interaction of event-driven and time-driven dynamics. This is motivated by the structure of many manufacturing environments where discrete entities (termed jobs) are processed through a network of workcenters so as to change their physical characteristics. Associated with each job is a temporal state subject to event-driven dynamics and a physical state subject to timedriven dynamics. Based on this framework, we formulate and analyze a class of optimal control problems for single-stage processes. First-order optimality conditions are derived and several properties of optimal state trajectories (sample paths) are identified which significantly simplify the task of obtaining explicit optimal control policies.
\end{abstract}

Index Terms-Hybrid system, nonsmooth optimization, optimal control.

\section{INTRODUCTION}

$\mathbf{T}$ HE term "hybrid" is used to characterize systems that combine time-driven and event-driven dynamics. The former are represented by differential (or difference) equations, while the latter may be described through various frameworks used for discrete event systems (DES), such as timed automata, max-plus equations, or Petri nets (see [5]). Broadly speaking, two categories of modeling frameworks have been proposed to study hybrid systems: Those that extend event-driven models to include time-driven dynamics; and those that extend the traditional time-driven models to include event-driven dynamics; for an overview, see [1]-[3], [12].

The hybrid system modeling framework we consider in this paper falls into the first category above. Although its scope is general, it is largely motivated by the structure of many manufacturing systems. In these systems, discrete entities (referred to as jobs) move through a network of workcenters which process the jobs so as to change their physical characteristics according to certain specifications. Associated with each job is a tem-

Manuscript received November 1, 1999; revised May 4, 2000 and June 21, 2000. Recommended by Editor A. Tits. The work of C. G. Cassandras was supported in part by the National Science Foundation under Grants EEC-9527422 and ACI-9873339, Air Force Office of Scientific Research under Grant F49620-98-1-0387, AFRL under Contract F30603-99-C-0057, and EPRI/DoD under Contract WO8333-03. The work of D. L. Pepyne was supported in part by EPRI/DoD under Contract WO8333-03, the U.S. Army under Contracts DAAL03-92-G-0115 and DAAH04-0148, Air Force Office of Scientific Research under Grant F49620-98-1-0387, and ONR under Contract N00014-98-10720.

C. G. Cassandras is with the Department of Manufacturing Engineering, Boston University, Boston, MA 02215 USA (e-mail: cgc@bu.edu).

D. L. Pepyne is with the Division of Engineering and Applied Sciences, Harvard University, Cambridge, MA 02138 USA (e-mail: pepyne@hrl.harvard.edu).

Y. Wardi is with the School of Electrical and Computer Engineering, Georgia Institute of Technology, Atlanta, GA 30332-0250 USA (e-mail: yorai.wardi@ee.gatech.edu).

Publisher Item Identifier S 0018-9286(01)02563-6. poral state and a physical state. The temporal state of a job evolves according to event-driven dynamics and includes information such as the waiting time or departure time of the job at the various workcenters. The physical state evolves according to time-driven dynamics modeled through differential (or difference) equations which, depending on the particular problem being studied, describe changes in such quantities as the temperature, size, weight, chemical composition, or some other measure of the "quality" of the job. The interaction of time-driven with event-driven dynamics leads to a natural tradeoff between temporal requirements on job completion times and physical requirements on the quality of the completed jobs. For example, while the physical state of a job can be made arbitrarily close to a desired "quality target," this usually comes at the expense of long processing times resulting in excessive inventory costs or violation of constraints on job completion deadlines. Our objective, therefore, is to formulate and solve optimal control problems associated with such tradeoffs.

The analysis and synthesis of optimal controllers for hybrid systems clearly requires a combination of techniques applicable to both time-driven and event-driven systems. In the latter case, although the parametric optimization of DES has been extensively researched (e.g., see [5] and the references therein), little progress has been reported in the area of optimal control, short of stochastic control methods (e.g., stochastic dynamic programming) that typically seek to optimize steady state (as opposed to transient) performance metrics. There are at least two important difficulties that have been blocking such progress: 1) the absence of a synchronizing clock that would permit the use of methodologies developed for classical time-driven systems (e.g., [4]); and 2) nondifferentiabilities in the event-driven state dynamics which limit the use of classical gradient-based techniques. Recently, however, it has been shown that these difficulties can be overcome in at least some problems [10], [17].

In this paper, we formulate and analyze a large class of optimal control problems for hybrid systems. We then show how, despite the difficulties mentioned above, the task of solving these problems is greatly simplified by exploiting the properties of the optimal state trajectories. In particular, an optimal state trajectory can be decomposed into fully decoupled segments, termed "busy periods." Moreover, each busy period can be further decomposed into "blocks" defined by certain jobs termed critical; identifying such jobs and their properties is a crucial part of the analysis and the key to developing effective algorithms for solving the optimal control problems. This observation was first made in [17] where a simpler and somewhat different problem than those included in the general framework of the present paper was analyzed without the use of any nonsmooth optimization techniques. 


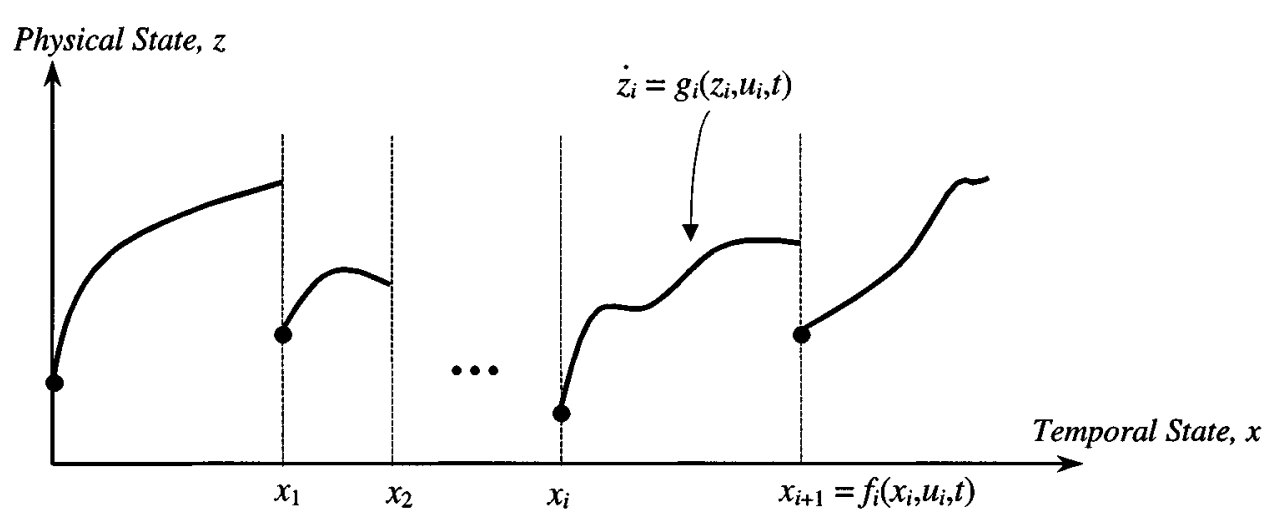

Fig. 1. Hybrid system framework.

The main contributions of our analysis are the following. First, we derive several conditions for identifying the critical jobs in an optimal sample path: One is a necessary and sufficient condition requiring minimal assumptions on the cost function; two more are sufficient conditions satisfied when the system has certain key properties. Second, for a class of problems with separable cost structure, we show that these key properties are indeed satisfied, which enables the development of efficient solution algorithms. We do not dwell on such algorithms in this paper, but refer the reader to related work reported elsewhere [8], [16], [18], [20], which is based on the results of this paper and is exclusively devoted to such algorithms and their analysis. Third, we also establish that for this class of problems the optimal solution is unique, despite the fact that the cost functions involved are not convex and not differentiable.

The paper is organized as follows. In Section II, we present a general framework for hybrid systems emphasizing the coupling between the time-driven dynamics of the system and the event-driven dynamics that govern switches in the system behavior. We also formulate an optimal control problem for the class of hybrid systems we consider. Section III analyzes the necessary conditions for optimality, introduces the nonsmooth optimization elements needed to handle the nondifferentiabilities involved, and concludes with a theorem that characterizes an optimal control sequence. Section IV presents several properties of the optimal solutions and introduces the concept of "critical jobs," crucial in the characterization of optimal sample paths. Conditions for identifying critical jobs are also derived in this section. In Section V, we analyze a class of problems with separable cost structure and show that a solution is unique even though the problem is not convex and not differentiable. We establish four important properties of the optimal sample paths, which facilitate the determination of critical jobs and hence the evaluation of the optimal solution.

\section{PRoblem Formulation}

The general hybrid system framework we consider is illustrated in Fig. 1. A system is initially at some physical state $\zeta_{1}$ at time $x_{0}$ and subsequently evolves according to the time-driven dynamics

$$
\dot{z}_{1}=g_{1}\left(z_{1}, u_{1}, t\right), \quad z_{1}\left(x_{0}\right)=\zeta_{1}
$$

where $u_{1}$ is a control (assumed scalar). At time $x_{1}$, a switch (event) takes place causing the physical state to become $z_{2}\left(x_{1}\right)=\zeta_{2}$. In general, we allow for $z_{2}\left(x_{1}\right) \neq z_{1}\left(x_{1}\right)$, and the physical state subsequently evolves according to new time-driven dynamics with this initial condition. The time of this switch, which we refer to as the temporal state of the system, depends on event-driven dynamics of the form

$$
x_{1}=f_{1}\left(x_{0}, z_{1}, u_{1}, t\right) .
$$

In general, after the $i$ th switch, the time-driven dynamics are given by

$$
\dot{z}_{i}=g_{i}\left(z_{i}, u_{i}, t\right), \quad z_{i}\left(x_{i}\right)=\zeta_{i}
$$

and the event-driven dynamics by

$$
x_{i}=f_{i}\left(x_{i-1}, z_{i}, u_{i}, t\right) .
$$

Note that the choice of control following the $i$ th switch affects both the physical state $z_{i}$ and the next temporal state $x_{i+1}$. Thus, the switches at times $x_{1}, x_{2}, \ldots$ are generally not exogenous events that dictate changes in the state dynamics, but rather temporal states intricately connected to the control of the system. We emphasize this fact since it is one of the crucial elements of a "hybrid" system. In some applications, the event-driven dynamics (1) may be viewed as exogenous switching times, substantially simplifying the analysis; this is not the case in the problems we tackle in what follows.

In the context of manufacturing systems, the switches in Fig. 1 correspond to jobs that we index by $i=1, \ldots, N$. We shall limit ourselves to a single-stage process modeled as a single-server queueing system. The objective is to process $N$ total jobs. The server processes one job at a time on a first-come first-served nonpreemptive basis (i.e., once a job begins service, the server cannot be interrupted, and will continue to work on it until the operation is completed). Jobs arriving when the server is busy wait in a queue whose capacity is $\geq N$. As job $i$ is being processed, its physical state, denoted by $z_{i} \in \mathbb{R}^{n}$, evolves according to time-driven dynamics of the general form

$$
\dot{z}_{i}=g_{i}\left(z_{i}, u_{i}, t\right), \quad z_{i}\left(\tau_{i}\right)=\zeta_{i}
$$

where $\tau_{i}$ is the time processing begins and $\zeta_{i}$ is the initial state at that time. The control variable $u_{i}$ (assumed here to be scalar 
and not time dependent for simplicity; however, see [11]) is used to attain a final desired physical state corresponding to a target "quality level." Specifically, if the service time for the $i$ th job is $s_{i}\left(u_{i}\right)$ and $\Gamma_{i}\left(u_{i}\right) \subset \mathbb{R}^{n}$ is a given set (e.g., a threshold above which $z_{i}$ satisfies a desired quality level), then the control $u_{i}$ is chosen to satisfy the stopping rule

$$
\begin{gathered}
s_{i}\left(u_{i}\right)=\min \left\{t \geq 0:\left[z_{i}\left(\tau_{i}+t\right)=\int_{\tau_{i}}^{\tau_{i}+t} g_{i}\left(z_{i}, u_{i}, \nu\right) d \nu\right.\right. \\
\left.\left.+\zeta_{i}\right] \in \Gamma_{i}\left(u_{i}\right)\right\}
\end{gathered}
$$

where $u_{i}$ takes a fixed constant value during the interval $\left[\tau_{i}, \tau_{i}+\right.$ $t$ ), and the "min" is assumed to exist. On the other hand, the temporal state of the $i$ th job is denoted by $x_{i}$ and represents the time when the job completes processing and departs from the system. Letting $a_{i}$ be the arrival time of the $i$ th job, the eventdriven dynamics describing the evolution of the temporal state are given by the following "max-plus" recursive equation:

$$
x_{i}=\max \left(x_{i-1}, a_{i}\right)+s_{i}\left(u_{i}\right)
$$

where we set $x_{0}=-\infty$ in which case $x_{1}=a_{1}+s_{1}\left(u_{1}\right)$ and the first job begins service as soon as it arrives. It is assumed that the job arrival sequence $\left\{a_{1}, \ldots, a_{N}\right\}$ is given (in some earlier work [10], arrival times were considered to be controllable). The recursive relationship (4) is known in queueing theory as the Lindley equation [5], and is the specific form of the event-driven dynamics (1) applicable to this particular hybrid system. In Fig. 1, an idle period corresponds to a situation where $a_{i+1}>x_{i}$, in which case there is an interval $\left[x_{i}, a_{i+1}\right]$ on the temporal state axis during which the physical state is undefined.

This system is hybrid is the sense that it combines the timedriven dynamics (2) with the event-driven dynamics (4), the two being coupled through the choice of the control sequence. The optimal control problem we consider has the general form

$$
\min _{u_{1}, \ldots, u_{N}} J=\sum_{i=1}^{N} L_{i}\left(x_{i}, u_{i}\right)
$$

subject to (2)-(4), where $L_{i}\left(x_{i}, u_{i}\right)$ is a cost function associated with job $i$. Note that this formulation does not require an explicit cost on the physical state $z_{i}$, since (3) ensures that each job satisfies a given quality requirement, i.e., $z_{i}\left(\tau_{i}+s_{i}\left(u_{i}\right)\right)=$ $z_{i}\left(x_{i}\right) \in \Gamma_{i}\left(u_{i}\right)$. This stopping rule defines a separate optimization problem, which must be solved to obtain the service time and its derivative. As an example, let $\Gamma_{i}\left(u_{i}\right)$ be a function of the control and suppose that the physical dynamics in (2) do not depend directly on the control. Thus, (2) and (3) assume the following respective forms: $\dot{z}_{i}=g_{i}\left(z_{i}\right)$ with initial condition $z_{i}\left(\tau_{i}\right)=\zeta_{i}$, and $s_{i}\left(u_{i}\right)=\min \left\{t \geq 0: z_{i}\left(\tau_{i}+t\right)=\Gamma_{i}\left(u_{i}\right)\right\}$. It can be seen, by directly applying variational principles, that

$$
\frac{d s_{i}}{d u_{i}}=\frac{1}{g_{i}\left(z_{i}\left(\tau_{i}+s_{i}\left(u_{i}\right)\right)\right)} \frac{d \Gamma_{i}}{d u}\left(u_{i}\right)
$$

assuming, of course, that the relevant derivatives exist.
The problem defined above appears similar to classical discrete-time optimal control problems commonly found in the literature (e.g., [4]) except for two issues. First, the index $i=$ $1, \ldots, N$ does not count time steps, but rather asynchronously departing jobs. Second, the presence of the "max" function in the state equation (4) prevents us from using standard gradientbased techniques, since it introduces a nondifferentiability at the point where $a_{i}=x_{i-1}$.

Regarding the first issue, although the absence of a synchronizing clock presents a difficulty encountered in all DES, note that the mathematical treatment of the recursive equation (4) is in fact no different than that of any other similar recursion where the index represents synchronized time steps as in classical discrete-time optimal control problems. Therefore, this issue is not really problematic. Regarding the second issue, previous work [17], [10] has shown that the nondifferentiability problem can be overcome in at least special cases of the problem formulated above, and that the "max" function exhibits certain useful structural properties that can be exploited to simplify the analysis and lead to efficient numerical solutions. For the more general class of problems considered here, we will invoke ideas and results from nondifferentiable calculus (e.g., [6]) to deal with the nondifferentiability issue.

Example: To illustrate the use of the framework and problem formulation presented above, we outline below an optimal control problem for steel heating/annealing manufacturing processes involving a furnace integrated with plant-wide planning and scheduling operations; full details and solutions based on the methods presented in this paper may be found in [7]. Individual steel "parts" (i.e., ingots or strips) undergo various operations to achieve certain metallurgical properties that define the "quality" of the finished products. In particular, the steel heating/annealing process is an important step which involves slowly heating and cooling strips to some desired temperatures. Before heating and cooling each roll of strips, a higher level controller determines the furnace reference temperature (more generally, a "furnace heating profile") which the strip should follow, as well as the amount of time that this strip is held in a furnace. Raw material, (e.g., a cold-rolled strip) is put on a pay-off reel on the entry side of the line and runs through with a certain line speed. The physical state of the $i$ th strip in this process is denoted by $z_{i}(t)$ and represents the temperature at each point of the strip as it evolves through the heating furnace. The strip temperature is basically dependent on the line speed $u_{i}$, which usually remains constant during the process, and the furnace reference temperature $F_{i}$, which is predesigned at a plant-wide planning level. The thermal process in the heating furnace can be represented by a nonlinear heat-transfer equation describing the dynamic response of each strip temperature so that the temporal change in heat energy at a particular location is equal to the transport heat energy plus the radiation heat energy [9] as follows:

$$
\frac{d z_{i}(t)}{d t}=-K_{1} u_{i}+K_{2}\left[F_{i}^{4}-z_{i}(t)^{4}\right], \quad t \geq t_{0}
$$

where

$$
K_{1}=\frac{F_{i}-z_{i}\left(t_{0}\right)}{L}, \quad K_{2}=\frac{2 \sigma_{s b} \phi_{s}}{60 d_{s} 10^{-3} \tau}
$$


and $L$ is the furnace length [m], $t_{0}$ is the heating start time, $\sigma_{s b}$ is the Stefan-Boltzmann constant $\left(=4.88 \times 10^{-8}\left[\mathrm{kcal} / \mathrm{m}^{2} \cdot \mathrm{h}\right.\right.$. $\left.\left.\operatorname{deg}^{4}\right]\right), \phi_{s}$ is the coefficient of radiative heat absorption $(0<$ $\phi_{s}<1$ ) (determined as 0.17 from actual data), $d_{s}$ is the strip specific heat $\left[\mathrm{kcal} / \mathrm{m}^{3} \cdot \mathrm{deg}\right]$, and $\tau$ is the strip thickness [mm].

Since (6) is in nonlinear differential form, it is hard to represent solutions in an explicit form. It turns out, however, that such solutions can be accurately approximated by exponential functions obtained as solutions of

$$
\frac{d z_{i}(t)}{d t}=\frac{1}{f\left(u_{i}\right)}\left(F_{i}-z_{i}(t)\right), \quad t \geq t_{0} .
$$

where $f\left(u_{i}\right)$ is an arbitrary function appropriately chosen to achieve a desired level of accuracy. In [7], $f\left(u_{i}\right)$ is taken to be a monotone increasing polynomial function of $u_{i}$, i.e., $f\left(u_{i}\right)=$ $\sum_{r=0}^{m} c_{k} u_{i}^{r}$ for some $m=1,2, \ldots$, an approximation successfully employed in practice [21].

Next, the temporal state of the $i$ th strip consists of two variables, $x_{i}$ and $y_{i}$, where $x_{i}$ represents the time when the job starts processing at the furnace and $y_{i}$ represents the time when the job completes processing and departs from the system. The need for two variables is due to the fact that we must distinguish between the starting time of the $(i+1)$ th job and the completion time of the $i$ th job (i.e., $x_{i+1} \neq y_{i}$ ), since each job is a continuous strip of a typical length, not a discrete entity. Letting $a_{i}$ be the arrival time of the $i$ th strip, the event-driven dynamics describing the evolution of these temporal states are given by

$$
\begin{aligned}
& x_{i}=\max \left(a_{i}, x_{i-1}\right)+s_{1}\left(u_{i}\right) \quad \text { and } \quad y_{i}=x_{i}+s_{2}\left(u_{i}\right) \\
& \text { subject to } u_{\min } \leq u_{i} \leq u_{\max }, \quad i=1, \ldots, N
\end{aligned}
$$

where $s_{1}\left(u_{i}\right)$ is the elapsed time for the whole body of the strip to enter the furnace, which is dependent on the length of the strip, and $s_{2}\left(u_{i}\right)$ is the processing time for each point of the strip to run through the furnace, which is dependent on the length of the furnace. In addition, $u_{\min }$ and $u_{\max }$ are the minimum and maximum allowable line speed respectively, and we assume that $x_{0}=-\infty$.

In this system, we consider two control objectives: 1) to reduce temperature errors with respect to the furnace reference temperature, and 2) to reduce the entire processing time for timely delivery using acceptable levels of line speed, $u_{i}$. Thus, the optimal control problem of interest is

$$
\min _{\left\{u_{1}, \ldots, u_{N}\right\}} J=\sum_{i=1}^{N}\left[\theta\left(u_{i}\right)+\phi\left(y_{i}\right)\right]
$$

subject to (7) and (8). The function $\phi\left(y_{i}\right)$ above is the cost related to jobs departing at time $y_{i}$. For example, $\phi\left(y_{i}\right)=\left(y_{i}-\right.$ $\left.d_{i}\right)^{2}$ is such that a job departing after the due date $d_{i}$ incurs a tardiness cost completing before its due date incurs an inventory (backlog) cost. The function $\theta\left(u_{i}\right)$ is selected so as to penalize the deviation of the $i$ th strip temperature from the reference temperature, $F_{i}$

$$
\begin{gathered}
\theta\left(u_{i}\right)=\left|F_{i}-z_{i}\left(L / u_{i}\right)\right|^{2}+\beta \int_{0}^{L / u_{i}}\left(F_{i}-z_{i}(t)\right)^{2} d t \\
i=1, \ldots, N
\end{gathered}
$$

where $L / u_{i}$ is the time each point of the strip stays in the furnace and $\beta$ is a weighting factor.

\section{NECESSARY CONDITIONS FOR OPTIMALITY}

We begin by invoking basic variational calculus techniques to study the minimization problem in (5) subject to (4). As in standard discrete-time optimal control problems, we define the augmented cost

$$
\begin{aligned}
\bar{J}(\mathbf{x}, \boldsymbol{\lambda}, \mathbf{u})=\sum_{i=1}^{N}\left\{L_{i}\left(x_{i}, u_{i}\right)\right. & \\
& \left.+\lambda_{i}\left[\max \left(x_{i-1}, a_{i}\right)+s_{i}\left(u_{i}\right)-x_{i}\right]\right\}
\end{aligned}
$$

where $\mathbf{x}$ and $\mathbf{u}$ are $N$-dimensional vectors for the temporal state and the control, and $\boldsymbol{\lambda}$ is an $N$-dimensional vector for the costate sequence used to adjoin the temporal dynamics in (4) to the cost in (5). Throughout the rest of our analysis, we will make the following assumptions.

Assumption A1: The one-step costs $L_{i}(\cdot, \cdot)$ and the service functions $s_{i}(\cdot)$ are continuously differentiable for all $i=1, \ldots, N$.

Assumption A2: The service functions $s_{i}(\cdot)$ are monotonically increasing for all $i=1, \ldots, N$.

Note that Assumption A2 can be replaced by service functions that are monotonically decreasing, depending on the nature of the control variables $u_{i}$, yielding dual results to those we will subsequently derive.

Ignoring for the moment the nondifferentiabilities associated with the "max" operation in (11), the standard first-order necessary conditions for optimality require that

$$
\frac{\partial \bar{J}}{\partial u_{i}}=0, \quad \frac{\partial \bar{J}}{\partial \lambda_{i}}=0, \quad \frac{\partial \bar{J}}{\partial x_{i}}=0 \quad \text { for all } i=1, \ldots, N \text {. }
$$

The first equation above gives the stationarity condition

$$
\frac{\partial L_{i}\left(x_{i}, u_{i}\right)}{\partial u_{i}}+\lambda_{i} \frac{d s_{i}\left(u_{i}\right)}{d u_{i}}=0 .
$$

The second equation in (12) recovers the state equation

$$
x_{i}=\max \left(a_{i}, x_{i-1}\right)+s_{i}\left(u_{i}\right)
$$

with initial condition $x_{0}=-\infty$. Finally, the third equation gives the costate equation

$$
\lambda_{i}=\frac{\partial L_{i}\left(x_{i}, u_{i}\right)}{\partial x_{i}}+\lambda_{i+1} \frac{d \max \left(x_{i}, a_{i+1}\right)}{d x_{i}}
$$

with boundary condition

$$
\lambda_{N}=\frac{\partial L_{N}\left(x_{N}, u_{N}\right)}{\partial x_{N}} .
$$

Equations (13)-(16) define a two-point boundary-value problem (TPBVP), whose solution provides a control sequence satisfying the necessary conditions for optimality. TPBVPs are notoriously hard; in our case, matters are further complicated by the presence of the "max" function in the costate equation (15). This function is Lipschitz continuous, differentiable in $x_{i}$ everywhere except at the single point where $x_{i}=a_{i+1}$ with

$$
\frac{d}{d x_{i}} \max \left(x_{i}, a_{i+1}\right)= \begin{cases}0, & \text { if } x_{i}<a_{i+1} \\ 1, & \text { if } x_{i}>a_{i+1}\end{cases}
$$


Moreover, at the point where $x_{i}=a_{i+1}$, the left and right derivatives clearly exist, given by 0 and 1 , respectively.

As the system operates, the sequence of arrival and departure times defines a state trajectory (or sample path). On any sample path, the points where $x_{i}=a_{i+1}$ acquire special significance, since they are responsible for the nondifferentiability of the "max" function in the costate equation (15). When such points are part of the optimal solution, the necessary conditions above cannot be used to establish optimality, and we must appeal to nonsmooth optimization theory, as described next. This will lead to the main result of this section, Theorem 3.1.

1) Nonsmooth Optimization: Given Assumption A1, the augmented cost $\bar{J}$, as the sum of Lipschitz functions, is itself a Lipschitz function. Such functions are continuous, but not everywhere differentiable. They are, however, differentiable almost everywhere (Radmacher's theorem). For Lipschitz functions, nonsmooth optimization gives the necessary conditions for optimality [6], [15]. In particular, suppose $f: \mathbb{R}^{n} \rightarrow \mathbb{R}$ is a locally Lipschitz continuous function of $u \in \mathbb{R}^{n}$, and let $S(u)$ denote the set of all sequences $\left\{u_{m}\right\}_{m=1}^{\infty} \subset \mathbb{R}^{n}$ that satisfy the following three conditions: i) $u_{m} \rightarrow u$ as $m \rightarrow \infty$, ii) The gradient $\nabla f\left(u_{m}\right)$ exists for all $m=1,2, \ldots$, iii) $\lim _{m \rightarrow \infty} \nabla f\left(u_{m}\right)=\phi$ exists. Then, the generalized gradient of $f$ at $u$ is denoted by $\partial f(u)$ and defined as the convex hull of all limits $\phi$ corresponding to every sequence $\left\{u_{m}\right\} \in S$. The generalized gradient has the following three fundamental properties [6]: i) $\partial f(u)$ is a nonempty, compact and convex set in $\mathbb{R}^{n}$, ii) $\partial f(u)$ is a singleton iff $f$ is continuously differentiable in some open set containing $u$, in which case $\partial f(u)=\{\nabla f(u)\}$, and iii) if $u$ is a local minimum of $f$, then $0 \in \partial f(u)$. The last property is an extension of the classical stationarity condition in (13), and becomes the first-order optimality condition in nonsmooth optimization.

As described above, the necessary condition for the optimization of nonsmooth Lipschitz functions is given in terms of $\partial f(u)$. Our task now, therefore, is to identify $\partial \bar{J}$. In order to do so, we introduce the following terminology that will be essential to all subsequent analysis:

Definition 1: An idle period is a time interval $\left(x_{k}, a_{k+1}\right]$ such that $x_{k}<a_{k+1}$ for any $k=1, \ldots, N-1$.

Definition 2: A busy period is a time interval $\left(a_{k}, x_{n}\right]$ defined by a subsequence $\{k, k+1, \ldots, n\}$ such that i) $x_{k-1}<$ $a_{k}$, ii) $x_{i} \geq a_{i+1}$ for all $i=k, \ldots, n-1$, and iii) $x_{n}<a_{n+1}$.

These terms are borrowed from classical queueing theory. An idle period is simply a time interval of strictly positive duration during which the server has no jobs to process, and a busy period is a time interval during which the server is processing jobs without any interruption caused by an empty input queue. A busy period, initiated at time $a_{k}$, must always follow an idle period, be followed by another idle period, and allow no other idle periods within it. We also set $a_{N+1}=\infty$ for consistency. The next term is introduced to capture an important special feature which we will show characterizes optimal sample paths for our problem.

Definition 3: A critical job with index $i$ is one that satisfies $x_{i}=a_{i+1}$.

Note that a critical job corresponds precisely to the situation where the "max" function is not differentiable in (15). More- over, note that a critical job cannot end a busy period; however, a busy period may contain one or more critical jobs.

In order to identify the busy period structure and the locations of critical jobs within a busy period, we associate with every job $i=1, \ldots, N$ the following two indices

$$
\begin{aligned}
n(i) & =\min \left\{n \geq i: x_{n}<a_{n+1}\right\}, \\
m(i) & =\min \left\{m \geq i: x_{m} \leq a_{m+1}\right\} .
\end{aligned}
$$

In words, $n(i)$ is the index of the last job in the busy period containing job $i$. Regarding $m(i)$, if job $i$ is critical or there are critical jobs between job $i$ and the end of its busy period, then $m(i)$ is the index of the first such critical job; in this case, $m(i)<n(i)$ and we have $x_{m(i)}=a_{m(i)+1}$. If, on the other hand, job $i$ is not critical and there are no critical jobs between job $i$ and the end of its busy period, then $m(i)$ is the index of the job that ends the busy period, i.e., $m(i)=n(i)$.

2) The $m(i)=n(i)$ Case: This is the simpler of the two cases, where job $i$ is not critical, there are no critical jobs between job $i$ and the end of its busy period, and we have $\max \left(x_{j}, a_{j+1}\right)=x_{j}>a_{j+1}$ for all $j=i, \ldots, n(i)-1$ and $\max \left(x_{n(i)}, a_{n(i)+1}\right)=a_{n(i)+1}>x_{n(i)}$. Therefore, all derivatives in the costate equation (15) exist and we get,

$$
\lambda_{i}=\sum_{j=i}^{n(i)} \frac{\partial L_{j}}{\partial x_{j}} .
$$

Then, the optimality condition (13) becomes

$$
\frac{\partial \bar{J}}{\partial u_{i}}=\frac{\partial L_{i}}{\partial u_{i}}+\frac{d s_{i}}{d u_{i}} \sum_{j=i}^{n(i)} \frac{\partial L_{j}}{\partial x_{j}}=0
$$

where we have omitted the arguments of the functions $L_{i}(\cdot)$ and $s_{i}(\cdot)$. Clearly, the same result holds when there are critical jobs in the busy period containing job $i$, as long as these critical jobs precede job $i$ in this busy period. In summary, we have established the following result.

Lemma 3.1: Under Assumption A1, if $m(i)=n(i)$, then $\bar{J}(\cdot)$ is locally continuously differentiable in $u_{i}$, and the optimality condition is

$$
\frac{\partial \bar{J}}{\partial u_{i}}=\frac{\partial L_{i}}{\partial u_{i}}+\frac{d s_{i}}{d u_{i}} \sum_{j=i}^{n(i)} \frac{\partial L_{j}}{\partial x_{j}}=0 .
$$

Letting $\partial \bar{J}_{i} \equiv \partial \bar{J} / \partial u_{i}$, it is clear that when $m(i)=n(i)$ we get

$$
\partial \bar{J}_{i}=\frac{\partial L_{i}}{\partial u_{i}}+\frac{d s_{i}}{d u_{i}} \sum_{j=i}^{n(i)} \frac{\partial L_{j}}{\partial x_{j}} .
$$

Thus, if critical jobs were to never occur on an optimal sample path [i.e., if $m(i)=n(i)$ for all $i=1, \ldots, N]$, then the function $\bar{J}$ would be differentiable at its minimum, the standard conditions for optimality would apply, and a numerical solution could be obtained by solving the TPBVP defined by (13)-(15).

3) The $m(i)<n(i)$ Case: Since, in general, $\bar{J}$ will exhibit the nondifferentiabilities associated with critical jobs, it is necessary to study next the case where $m(i)<n(i)$. For any such job $i$ on an optimal sample path, we have $\max \left(x_{m(i)}, a_{m(i)+1}\right)$ 
$=x_{m(i)}=a_{m(i)+1}$ and the corresponding derivative in the costate equation (15) does not exist. Hence, the derivative $\partial \bar{J} / \partial u_{i}$ also fails to exist. To obtain the generalized gradient in this case we proceed as follows. First, since jobs $i$ and $m(i)$ are in the same busy period and $m(i) \geq i$, we have

$$
x_{m(i)}=\max \left(x_{i-1}, a_{i}\right)+s_{i}\left(u_{i}\right)+\sum_{j=i+1}^{m(i)} s_{j}\left(u_{j}\right)
$$

where the "max" accounts for the fact that job $i$ may be the first in the busy period. Through (21) we see that the control for job $i$ affects the departure time of job $m(i)$. Now suppose that we fix all controls at their optimal values and perturb $u_{i}$. Recalling (17), the following one-sided derivatives exist:

$$
\begin{aligned}
& \lim _{x_{m(i)} \uparrow a_{m(i)+1}} \frac{d}{d x_{m(i)}} \max \left(x_{m(i)}, a_{m(i)+1}\right)=0 \\
& \lim _{x_{m(i)} \downarrow a_{m(i)+1}} \frac{d}{d x_{m(i)}} \max \left(x_{m(i)}, a_{m(i)+1}\right)=1 .
\end{aligned}
$$

Conceptually, the first limit in (22) corresponds to the process of changing $u_{i}$ so that $x_{m(i)}$ increases toward a fixed $a_{m(i)+1}$. Similarly, the second limit corresponds to the process of changing $u_{i}$ so that $x_{m(i)}$ decreases toward $a_{m(i)+1}$ and the same is true for all other critical jobs between $m(i)$ and $n(i)$.

Looking at (15), note that

$$
\frac{d \max \left(x_{j}, a_{j+1}\right)}{d x_{j}}=1 \quad \text { for all } j=i, \ldots, m(i)-1 .
$$

Thus, combining (11) and (15), we get

$$
\begin{aligned}
\frac{\partial \bar{J}}{\partial u_{i}}= & \frac{\partial L_{i}}{\partial u_{i}}+\frac{d s_{i}}{d u_{i}} \\
& \cdot\left[\sum_{j=i}^{m(i)} \frac{\partial L_{j}}{\partial x_{j}}+\lambda_{m(i)+1} \frac{d \max \left(x_{m(i)}, a_{m(i)+1}\right)}{d x_{m(i)}}\right] .
\end{aligned}
$$

By Assumption A2 and (21), $x_{m(i)}$ is monotonically increasing in $u(i)$ and, using (22), the preceding equation leads to the onesided derivative

$$
\left(\frac{\partial \bar{J}}{\partial u_{i}}\right)^{-}=\frac{\partial L_{i}}{\partial u_{i}}+\frac{d s_{i}}{d u_{i}} \sum_{j=i}^{m(i)} \frac{\partial L_{j}}{\partial x_{j}}
$$

Similarly, we obtain

$$
\left(\frac{\partial \bar{J}}{\partial u_{i}}\right)^{+}=\frac{\partial L_{i}}{\partial u_{i}}+\frac{d s_{i}}{d u_{i}} \sum_{j=i}^{n(i)} \frac{\partial L_{j}}{\partial x_{j}}
$$

regardless of whether one or more critical jobs are present between $i$ and $n(i)$. For simplicity, we shall use the notation $\xi_{i}^{-}$ and $\xi_{i}^{+}$to denote the left and right derivatives above, i.e., set

$$
\xi_{i}^{-}=\left(\frac{\partial \bar{J}}{\partial u_{i}}\right)^{-}, \quad \xi_{i}^{+}=\left(\frac{\partial \bar{J}}{\partial u_{i}}\right)^{+}
$$

Regarding $\xi_{i}^{-}$and $\xi_{i}^{+}$, we can easily establish the following.
Lemma 3.2: Under Assumptions $\mathbf{A 1}$ and $\mathbf{A 2}$, for every $i=$ $1, \ldots, N$

$$
\xi_{i}^{+}=\xi_{i}^{-}+\frac{d s_{i}}{d u_{i}} \sum_{j=m(i)+1}^{n(i)} \frac{\partial L_{j}}{\partial x_{j}} .
$$

Proof: From (23) and (24)

$$
\begin{aligned}
\xi_{i}^{+} & =\frac{\partial L_{i}}{\partial u_{i}}+\frac{d s_{i}}{d u_{i}} \sum_{j=i}^{n(i)} \frac{\partial L_{j}}{\partial x_{j}} \\
& =\frac{\partial L_{i}}{\partial u_{i}}+\frac{d s_{i}}{d u_{i}} \sum_{j=i}^{m(i)} \frac{\partial L_{j}}{\partial x_{j}}+\frac{d s_{i}}{d u_{i}} \sum_{j=m(i)+1}^{n(i)} \frac{\partial L_{j}}{\partial x_{j}} \\
& =\xi_{i}^{-}+\frac{d s_{i}}{d u_{i}} \sum_{j=m(i)+1}^{n(i)} \frac{\partial L_{j}}{\partial x_{j}}
\end{aligned}
$$

giving (26).

Recalling the definition of $\partial \bar{J}$, it is easy to see that when $m(i)<n(i)$ we have

$$
\partial \bar{J}_{i}=\left[\min \left(\xi_{i}^{-}, \xi_{i}^{+}\right), \max \left(\xi_{i}^{-}, \xi_{i}^{+}\right)\right] \subset \mathbb{R} .
$$

Notice that when $m(i)=n(i)$, we get $\xi_{i}^{-}=\xi_{i}^{+}$, in which case the set $\partial \bar{J}_{i}$ defined by the closed interval above is a singleton equal to the gradient $\partial \bar{J} / \partial u_{i}$ as required. To summarize, we present next the main result of this section:

Theorem 3.1: Under Assumptions A1 and A2, an optimal control $u_{i}, i=1, \ldots, N$ satisfies the following conditions:

1) $0 \in \partial \bar{J}_{i}=\left[\min \left(\xi_{i}^{-}, \xi_{i}^{+}\right), \max \left(\xi_{i}^{-}, \xi_{i}^{+}\right)\right] \subset \mathbb{R}$, where

$$
\begin{aligned}
& \xi_{i}^{-}=\frac{\partial L_{i}}{\partial u_{i}}+\frac{d s_{i}}{d u_{i}} \sum_{j=i}^{m(i)} \frac{\partial L_{j}}{\partial x_{j}}, \quad \xi_{i}^{+}=\frac{\partial L_{i}}{\partial u_{i}}+\frac{d s_{i}}{d u_{i}} \sum_{j=i}^{n(i)} \frac{\partial L_{j}}{\partial x_{j}} \\
& m(i)=\min \left\{m \geq i: x_{m} \leq a_{m+1}\right\}, \\
& n(i)=\min \left\{n \geq i: x_{n}<a_{n+1}\right\} ; \\
& \text { 2) } x_{i}=\max \left(a_{i}, x_{i-1}\right)+s_{i}\left(u_{i}\right) x_{0}=-\infty .
\end{aligned}
$$

Proof: The proof follows directly from the necessary condition of nonsmooth optimization, that is, the requirement that $0 \in \partial \bar{J}_{i}$, and from Lemma 3.1 and (23)-(25).

Remark 3.1: Recalling Lemma 3.1, we see that when $m(i)=$ $n(i)$, i.e., when job $i$ is not critical and there are no critical jobs between job $i$ and the end of its busy period, then the first condition of the theorem simply requires that $\xi_{i}^{-}=\xi_{i}^{+}=0$.

Remark 3.2: For typical $L_{i}(\cdot, \cdot)$ and $s_{i}(\cdot)$, neither $\xi_{i}^{-}=0$ nor $\xi_{i}^{+}=0$ when $m(i)<n(i)$, i.e., in general, zero is not an endpoint of the interval defining $\partial \bar{J}_{i}$. Hence, when $m(i)<n(i)$ the first condition of the theorem requires that these quantities have opposite signs, i.e., $\left(\xi_{i}^{-}\right) \cdot\left(\xi_{i}^{+}\right)<0$. In general, however, $\left(\xi_{i}^{-}\right) \cdot\left(\xi_{i}^{+}\right) \leq 0$

We should also point out that the use of the generalized gradient is not indispensable for the solution of the problems considered here. In earlier work [17], for example, a specific hybrid system optimal control problem that belongs to the class of problems being studied in this paper was solved using a definition of the derivative of the "max" function that allows its value to be some arbitrary $\Delta_{i}$ such that $0 \leq \Delta_{i} \leq 1$ whenever $x_{i}=$ 
$a_{i+1}$. Finally, note that the problems can also be tackled through constrained nonlinear programming techniques; the computational burden in this case, however, is prohibitive for values of $N$ other than very small ones, and this serves to motivate the analysis that follows.

\section{Properties of Optimal Solutions}

Based on the necessary conditions for optimality in Theorem 3.1 , in this section we present some fundamental properties of optimal sample paths.

\section{A. Decoupling Properties}

The presence of the "max" function appearing in the state and costate equations leads to decoupling properties which decompose sample paths into independent segments. The first such property is a consequence of the "regenerative" nature of the state trajectory. Because of the "max" function in the state equation, information is not propagated in the forward direction across idle periods. In addition, because of the " $m a x$ " function in the costate equation, information does not propagate in the backward direction across idle periods. As a result, we obtain what we call idle period decoupling.

Lemma 4.1: Consider a busy period defined by $\{k, \ldots, n(k)\}$ and let $i \in\{k, \ldots, n(k)\}$. The optimal control $u_{i}^{*}$ depends only on $a_{k}, \ldots, a_{n(k)}$ (it does not depend on the arrival times of jobs in any other busy period).

Proof: In view of Theorem 3.1, observe that the state equation does not propagate information in the forward direction across the idle period that precedes the busy period containing job $i$, i.e.,

$$
\begin{aligned}
x_{k-1} & <a_{k} \Rightarrow \max \left(x_{k-1}, a_{k}\right)=a_{k} \Rightarrow x_{i} \\
& =a_{k}+s_{k}\left(u_{k}\right)+\cdots+s_{i}\left(u_{i}\right) .
\end{aligned}
$$

Hence, the control for job $i$ does not depend on the arrival times of jobs in earlier busy periods. Moreover, the costate equation does not propagate information in the backward direction across the idle period that follows the busy period containing job $i$, i.e.,

$$
\begin{aligned}
x_{n(k)} & <a_{n(k)+1} \Rightarrow \frac{d \max \left(x_{n(k)}, a_{n(k)+1}\right)}{d x_{n(k)}}=0 \Rightarrow \xi_{i}^{+} \\
& =\frac{\partial L_{i}}{\partial u_{i}}+\frac{d s_{i}}{d u_{i}} \sum_{j=i}^{n(k)} \frac{\partial L_{j}}{\partial x_{j}}
\end{aligned}
$$

and the same is true for $\xi_{i}^{-}$in (24), since $m(i) \leq n(k)$. Since, by Theorem 3.1, the optimal control $u_{i}^{*}$ is determined by $\xi_{i}^{-}$, $\xi_{i}^{+}$, it follows that it does not depend on the arrival times of jobs in subsequent busy periods.

Because of idle period decoupling, the controls for individual busy periods can be determined independently of each other. Therefore, idle period decoupling decomposes a large TPBVP consisting of $N$ jobs into several smaller subproblems, one for each busy period. Of course, since the identification of busy periods themselves is not a simple matter, this only partially simplifies the solution approach. Nonetheless, this decomposition can be used to develop efficient numerical algorithms (see [8], [16], [18], and [20]). Moreover, it is also useful in the theoretical analysis of the optimal sample path, since it allows us to study its properties by analyzing a single isolated busy period.

Whereas idle periods decompose the problem into a collection of independent busy periods, critical jobs further decompose the problem by partitioning busy periods into collections of blocks, where a block is defined as follows.

Definition 4: Consider a busy period consisting of jobs $\{k, \ldots, n(k)\}$. A block is a subset $\{j, \ldots, m(j)\} \subseteq$ $\{k, \ldots, n(k)\}$ such that

1) for all $i \in\{j, \ldots, m(j)\}, m(i)=m(j)$;

2) for all $i \in\{k, \ldots, n(k)\}$ and $i \notin\{j, \ldots, m(j)\}$, $m(i) \neq m(j)$.

In other words, any busy period on an optimal sample path can be partitioned into blocks, where the first block begins with the first job and ends with the first critical job (if any). The second block begins with the job that follows the first critical job and ends with the second critical job, and the last block ends with the last job in the busy period (therefore, it never contains a critical job). Clearly, if a busy period consists of $L$ blocks, then there are $L-1$ critical jobs in this busy period. Moreover, every block starts with an arrival time $a_{j}$ such that $j=k$ for the first block and $a_{j}=x_{j-1}$ for the remaining blocks. The notion of blocks leads to what we call the partial coupling property.

Lemma 4.2: Consider a block defined by $\{j, \ldots, m(j)\}$ and let $i \in\{j, \ldots, m(j)\}$. The optimal control $u_{i}^{*}$ depends only on $a_{j}$ and $a_{m(j)+1}$ (it does not depend on any other arrival times).

Proof: Consider a busy period containing at least one critical job. Notice that the state equation does not propagate across critical jobs, i.e., $x_{i}=a_{j}+s_{j}\left(u_{j}\right)+\cdots+s_{i}\left(u_{i}\right)$. Hence, the optimal controls for jobs $\{j, \ldots, m(j)\}$ can be obtained by solving the following optimization problem:

$$
\min _{u_{j}, \ldots, u_{m(j)}} \sum_{i=j}^{m(j)} L_{i}\left(x_{i}, u_{i}\right)
$$

subject to $x_{i}=a_{j}+s_{j}\left(u_{j}\right)+\cdots+s_{i}\left(u_{i}\right)$ for all $i=j, \ldots, m(j)$ and terminal constraint $x_{m(j)}=a_{m(j)+1}$ provided this is not the last block in the busy period; if it is the last block, then the constraint is $x_{m(j)}<a_{m(j)+1}$. Thus, the solution depends only on $a_{j}$ and $a_{m(j)+1}$ (and of course $m(j))$.

Because of partial coupling, the controls for those jobs that follow a critical one are independent of the controls for the jobs that precede it. This property forms the basis of algorithms one can develop to explicitly solve the problem under study, as further discussed in what follows.

\section{B. Critical Job Characterization}

Critical jobs play a crucial role in obtaining explicit solutions for the optimal control problem under consideration. This is obvious from the decoupling properties of the previous section; if we could easily identify the various indices $m(i)$ and $n(i)$ for each job $i=1, \ldots, N$, then we could solve the problem by solving a collection of TPBVP's, one for each block. Some of these TPBVP's would have a terminal constraint on the final state to force the departure time of the last job in the block to equal the arrival time of the job that begins the next block, while 
others would not have terminal constraints when the block ends a busy period.

Although the possibility of critical jobs depends on the specific forms of the one-step costs $L_{i}(\cdot, \cdot)$ and the service functions $s_{i}(\cdot)$, we point out that for most problems of practical interest the occurrence of critical jobs is not an "unusual" or pathological case, but an integral part of a typical optimal sample path, as demonstrated in our earlier work [17], [10].

Before proceeding, we shall make one additional assumption regarding the nature of the functions $L_{i}(\cdot, \cdot)$ and $s_{i}(\cdot)$ :

Assumption A3: The one-step costs $L_{i}(\cdot)$ are strictly convex functions and the service functions $s_{i}(\cdot)$ are convex functions of their arguments for all $i=1, \ldots, N$.

Let us also define

$$
\xi_{i, k}=\frac{\partial L_{i}}{\partial u_{i}}+\frac{d s_{i}}{d u_{i}} \sum_{j=i}^{k} \frac{\partial L_{j}}{\partial x_{j}}
$$

and note that, by definition (25), we have

$$
\xi_{i, m(i)}=\xi_{i}^{-} \quad \text { and } \quad \xi_{i, n(i)}=\xi_{i}^{+}
$$

Thus, if job $i$ is critical, then $i=m(i)$ and $\xi_{i, i}=\xi_{i}^{-}$, an observation that turns out to be very useful in our analysis.

The following theorem gives necessary and sufficient conditions that must be satisfied by a critical job (a similar result can be established if Assumption A2 is changed to consider monotonically decreasing service functions).

Theorem 4.1: Under Assumptions A1-A3, job $i$ is critical on an optimal sample path if and only if

1) $\xi_{i, i}\left(u_{i}^{*}-\Delta\right)=\xi_{i, n(i)}\left(u_{i}^{*}-\Delta\right)$;

2) $\xi_{i, i}\left(u_{i}^{*}+\Delta\right) \cdot \xi_{i, n(i)}\left(u_{i}^{*}+\Delta\right)<0$; where $u_{i}^{*}$ is the optimal control for job $i, n(i)$ is the index of the job that ends the busy period containing job $i$ under the control $u_{i}=u_{i}^{*}-\Delta$ in (1) and $u_{i}=u_{i}^{*}+\Delta$ in (2), and $\Delta>0$ is some arbitrarily small perturbation satisfying $\xi_{i, i}\left(u_{i}^{*}+\Delta\right) \neq 0$, $\xi_{i, n(i)}\left(u_{i}^{*}+\Delta\right) \neq 0$.

Proof: Throughout the proof, recall that the index $n(i)$ depends on the control sequence, although for notational simplicity this dependence is not explicitly shown.

First, suppose job $i$ is critical on an optimal sample path. We will then show that conditions (1) and (2) hold. Under the optimal control $u_{i}^{*}$ we have $x_{i}=a_{i+1}$. By Assumption A2, $s_{i}\left(u_{i}\right)$ is increasing in $u_{i}$; therefore, decreasing the control by $\Delta>0$ decreases the service time for job $i$. This introduces an idle period between jobs $i$ and $i+1$, in which case $n(i)=i$, and condition (1) immediately follows. Regarding condition (2), since job $i$ is critical, we have $i=m(i)<n(i)$, in which case Theorem 3.1 requires that $0 \in \partial \bar{J}=\left[\min \left(\xi_{i}^{-}, \xi_{i}^{+}\right), \max \left(\xi_{i}^{-}, \xi_{i}^{+}\right)\right]$ $=\left[\min \left(\xi_{i, m(i)}, \xi_{i, n(i)}\right), \max \left(\xi_{i, m(i)}, \xi_{i, n(i)}\right)\right]$, where we have used (28). This requires that $\xi_{i, i}$ and $\xi_{i, n(i)}$ have opposite sign. Hence, condition (2) holds for $\Delta=0$. It also holds for arbitrarily small $\Delta>0$ since i) by $\mathbf{A} \mathbf{1}, \xi_{i, k}$ is a continuous function of $u_{i}$, and ii) for arbitrarily small positive perturbations in the control, the index $n(i)$ remains fixed [i.e., job $n(i)$ still ends the busy period].

Conversely, if conditions (1) and (2) hold for some job $i$ on an optimal sample path, we shall show that $i$ is critical. Under A3, and in view of (28), condition (1) implies that $i=n(i)$. Therefore, decreasing the control for job $i$ by $\Delta$ decreases its departure time (from A2) and the perturbed sample path contains an idle period between job $i$ and job $i+1$ [since $i=n(i)$ is now the last job in the busy period]. This implies that on the optimal path (prior to the arbitrarily small perturbation $\Delta$ in $u_{i}$ ) either i) job $i$ is critical, or ii) job $i$ is the last job in its busy period. If iii) is the case, then on the optimal sample path job $i$ is followed by an idle period of finite duration. Hence, for small positive perturbations in the control, job $i$ is still the last in its busy period, i.e., $i=n(i)$ in such a perturbed path, and $\xi_{i, i}\left(u_{i}^{*}+\Delta\right)$ $=\xi_{i, n(i)}\left(u_{i}^{*}+\Delta\right)$. However, this contradicts the assumption that condition (2) holds. Hence, job $i$ cannot be the last job in its busy period and case i) must hold, i.e., job $i$ is indeed critical, and the proof is complete.

The importance of this result manifests itself in algorithms we can develop (see [17], [18]) for the numerical solution of the optimal control problem. By iteratively evaluating the quantities $\xi_{i, i}(\cdot)$ and $\xi_{i, n(i)}(\cdot)$, the two conditions in the theorem allow us to identify critical jobs (with arbitrary accuracy dependent on $\Delta$ ). This, as previously argued, makes it possible to decompose a sample path into blocks which can be separately analyzed to determine the optimal control sequence within each one, a significant computational simplification when it comes to a TPBVP.

Noncritical Departures and Their Properties: The remainder of this section is devoted to further identifying conditions that lead to critical jobs and provide insight to their importance in this class of problems. Let us consider a busy period containing $B$ jobs on an optimal sample path. Because of idle period decoupling (Lemma 4.1), there is no loss of generality if we index the first job in the busy period as job 1 [and relabel accordingly all cost components $L_{i}(\cdot, \cdot)$, so that $i=1, \ldots, B]$. Then, $n(1)=B$ is the number of jobs in this busy period. When the busy period does not contain any critical jobs, i.e., when $m(1)=n(1)=B$, let the optimal departure times be denoted by $\left\{x_{1, B}, \ldots, x_{B, B}\right\}$. Thus, in the notation $x_{i, B}, i$ denotes the index of the job within the busy period and $B$ is the total number of jobs in the busy period.

Definition 5: The optimal departure times when there are no critical jobs in a busy period defined by $\{1, \ldots, B\}$ are denoted by $\left\{x_{1, B}, \ldots, x_{B, B}\right\}$ and referred to as noncritical departures. The corresponding optimal controls are denoted by $\left\{u_{1, B}, \ldots, u_{B, B}\right\}$ and referred to as noncritical controls.

An important property of the noncritical departures, shown next, is that they can all be precomputed offline for any given positive integer $B$ and any specified arrival time for the first job in the busy period, $a_{1}$. Thus, strictly speaking we should write $x_{i, B}\left(a_{1}\right)$, but omit the dependence on $a_{1}$ for simplicity. Observe that any $B$ jobs $\{j, \ldots, j+B-1\}$ may be selected, re-indexed as $\{1, \ldots, B\}$, and then assigned values $\left\{x_{1, B}, \ldots, x_{B, B}\right\}$. In other words, any set of $B$ jobs may be used in a simple "thought experiment" that allows us to evaluate their departure times as if these formed a busy period with no critical jobs.

Lemma 4.3: The noncritical departures $\left\{x_{1, B}, \ldots, x_{B, B}\right\}$ depend only on $a_{1}$ and $B$.

Proof: Consider a busy period consisting of jobs $\{1, \ldots, B\}$ on an optimal sample path and assume that none 
of these jobs is critical. If this is the case, then the noncritical departures are optimal by definition. By Theorem 3.1, the optimal controls corresponding to these noncritical departures must satisfy i) the state equation (14), and ii) the condition $0 \in \partial \bar{J}_{i}$, where $\partial \bar{J}_{i}$ is a closed interval defined by $\xi_{i}^{-}$and $\xi_{i}^{+}$. From i), the state equation for time associated with any job $i \in\{1, \ldots, B\}$ gives

$$
x_{i, B}=a_{1}+s_{1}\left(u_{1}\right)+\cdots+s_{i}\left(u_{i}\right), \quad i=1, \ldots, B .
$$

From ii), since there are no critical jobs during this busy period, $m(i)=n(i)$, and

$\xi_{i}^{-}=\xi_{i}^{+}=\frac{\partial L_{i}}{\partial u_{i}}+\frac{d s_{i}}{d u_{i}} \sum_{j=i}^{B} \frac{\partial L_{j}}{\partial x_{j, B}}=0, \quad i=1, \ldots, B$.

The above expressions depend only on $a_{1}$ and $B$, since the functions $L_{i}\left(x_{i}, u_{i}\right)$ and $s_{i}\left(u_{i}\right)$ are independent of the arrival sequence $\left\{a_{1}, \ldots, a_{N}\right\}$. Therefore, the controls $\left\{u_{1}, B, \ldots, u_{B}, B\right\}$ obtained by solving (29) and (30) depend only on $a_{1}$ and $B$, and, consequently, the noncritical departures $\left\{x_{1, B}, \ldots, x_{B, B}\right\}$ obtained through (29) depend only on $a_{1}$ and $B$.

Note that an alternative definition of the noncritical departures is that they are the unique solution obtained from (29) and (30).

The next two lemmas provide characterizations of critical jobs on an optimal sample path based on the relative ordering of the known arrival sequence and the noncritical departures which, we reiterate, may be precomputed for any given arrival time $a_{1}$ and positive integer $B$. These characterizations are derived under four conditions, referred to as properties P1-P4 below. The significance of these properties will become apparent in the next section where we show that a large class of problems indeed satisfies all four conditions. In what follows, given a busy period consisting of jobs $1, \ldots, B$ on an optimal sample path, we shall denote the optimal departure times for the jobs in this busy period by $x_{1}, \ldots, x_{B}$.

Property P1 (Uniqueness): The optimal control sequence is unique.

Property $\mathbf{P 2}$ (Monotonicity in B): For a given arrival time $a_{1}$ that starts a busy period, the noncritical departure times are monotonically decreasing in the number of jobs in the busy period, i.e., $x_{i, \bar{B}} \leq x_{i, B}$ for all $i=1, \ldots, B$ and $B \leq \bar{B}$.

Property $\mathbf{P 3}$ (Lower Bounds for Optimal Departures): In a busy period consisting of jobs indexed $\{1, \ldots, B\}$, the noncritical departure times lower bound the optimal departure times, i.e., $x_{i, B} \leq x_{i}$ for all $i=1, \ldots, B$.

Property $\mathbf{P 4}$ (Upper Bounds for Optimal Departures): In a block consisting of jobs $\{1, \ldots, L\}$, the noncritical departure times upper bound the optimal departure times, i.e., $x_{i} \leq x_{i, L}$ for all $i=1, \ldots, L$ (Note: In this case, $a_{1}$ refers to the arrival time of the first job in the block, and not necessarily the arrival time of the first job in the busy period that contains this block.)

Lemma 4.4: Consider a busy period on an optimal sample path consisting of jobs indexed $\{1, \ldots, B\}$ and let $\bar{N} \leq N$ denote the number of jobs remaining to be processed starting with job 1. Under P1-P4, if there exists some $L \leq \bar{N}$ such that $a_{i+1}<x_{i, \bar{N}}$ for all $i=1, \ldots, L-1$ and $x_{L, L+1} \leq a_{L+1} \leq$ $x_{L, L}$, then job $L$ is critical.

Proof: We proceed by contradiction and show that neither $x_{L}<a_{L+1}$ nor $x_{L}>a_{L+1}$ can be optimal, which implies that $x_{L}=a_{L+1}$, i.e., $L$ is critical.

First, suppose $x_{L}<a_{L+1}$. Then, there is an idle period between jobs $L$ and $L+1$. By P2, $x_{i, \bar{N}} \leq x_{i, L}$. Since we assume $a_{i+1}<x_{i, \bar{N}}$ for all $i=1, \ldots, L-1$, we also have $a_{i+1}<x_{i, L}$ for all $i=1, \ldots, L-1$. Recalling Definition 2, uniqueness of the optimal solution (Property P1) implies a busy period consisting of jobs $i=1, \ldots, L$ in which there are no critical jobs. If this is the case, then the noncritical departures $x_{i, L}$ are by definition optimal for all $i=1, \ldots, L$. However, $x_{L}=x_{L, L}<a_{L+1}$ contradicts the assumption that $a_{L+1} \leq x_{L, L}$.

On the other hand, suppose $x_{L}>a_{L+1}$. Then, jobs $L$ and $L+1$ are in the same block. Moreover, we show next that this must be the first block in the busy period (i.e., the one that begins with job 1 and starts at time $a_{1}$ ). In particular, suppose the first block in the busy period ends with some job $j \geq 1$. Then, $x_{j, \bar{N}} \leq x_{j, B}$ by $\mathbf{P 2} ; x_{j, B} \leq x_{j}$ by $\mathbf{P 3}$; and $x_{j} \leq a_{j+1}$, since $j$ ends the block, giving

$$
x_{j, \bar{N}} \leq x_{j, B} \leq x_{j} \leq a_{j+1}
$$

where $B(j \leq B \leq \bar{N})$ is the number of jobs in the busy period containing job $j$. Since job $j$ ends a block, either i) job $j$ is not critical, or ii) job $j$ is critical. If i) is true, then $j$ also ends the busy period, i.e., $j=B \leq \bar{N}$ and $x_{j, j}=x_{j}$ since noncritical departures must be optimal. Using P2, we then get

$$
x_{j, \bar{N}} \leq x_{j, j}=x_{j}<a_{j+1} .
$$

If ii) is true, then $j<B \leq \bar{N}$ and

$$
x_{j, \bar{N}} \leq x_{j, B} \leq x_{j}=a_{j+1}
$$

where the first inequality follows from $\mathbf{P 2}$ and the second from P3. Now, suppose $j<L+1$. Then, in either case above, we are led to a contradiction of the assumption that $a_{i+1}<x_{i, \bar{N}}$ for all $i=1, \ldots, L-1$ and that $x_{L}>a_{L+1}$. Therefore, we must have $j \geq L+1$, that is, $L$ and $L+1$ are both members of the first block of a busy period, and this block contains $j \geq L+1$ jobs. However, if job $L$ is in a block that contains $j \geq L+1$ jobs then, using $\mathbf{P 4}$ and $\mathbf{P 2}$, we get

$$
a_{L+1}<x_{L} \leq x_{L, j} \leq x_{L, L+1}
$$

which contradicts the assumption that $x_{L, L+1} \leq a_{L+1}$. We have, therefore, established that $x_{L}=a_{L+1}$, i.e., job $L$ must be critical.

The conditions of Lemma 4.4 are only sufficient, i.e., there are other conditions that will result in critical jobs. The next result gives a different, more general, characterization of the conditions satisfied by critical jobs.

Lemma 4.5: Consider a busy period on an optimal sample path consisting of jobs indexed $\{1, \ldots, B\}$. Under P1-P4, if $x_{j, B} \leq a_{j+1}$ for any $j=1, \ldots, B-1$, then the busy period contains at least one critical job. Moreover, the first critical job in the busy period satisfies $x_{j, B} \leq a_{j+1} \leq x_{j, j}$. 

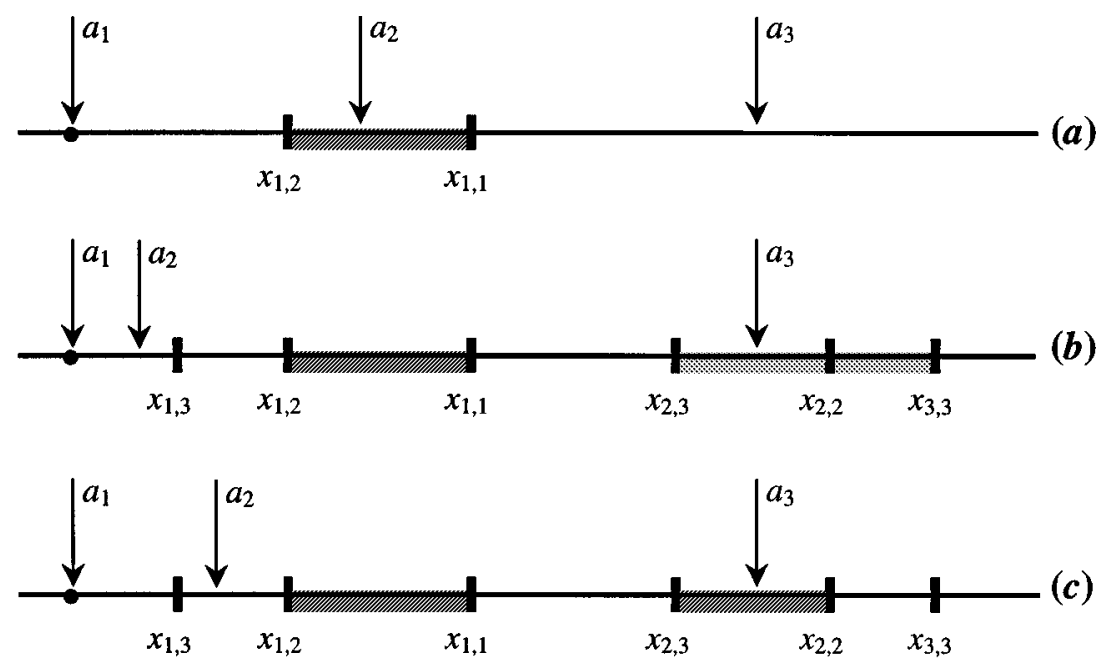

Fig. 2. Critical intervals for an example with $N=3$.

Proof: First, by Definition 2, because the busy period contains $B$ jobs, we must have $a_{i+1} \leq x_{i}$ for all $i=1, \ldots, B-1$.

To prove the first part of the lemma, suppose that $x_{j, B} \leq$ $a_{j+1}$ for one or more jobs $j=1, \ldots, B-1$, but the busy period does not contain any critical jobs. If the busy period does not contain any critical jobs, then the noncritical departures are optimal by uniqueness (Property P1), i.e., $x_{j}=x_{j, B}$ for all $j=$ $1, \ldots, B$. But, $x_{j}=x_{j, B}>a_{j+1}$ contradicts the assumption that $x_{j, B} \leq a_{j+1}$, implying that the busy period must contain at least one critical job.

Regarding the second part of the lemma, first note that $\mathbf{P 2}$ guarantees that indeed $x_{j, B} \leq x_{j, j}$. Then, if job $j$ is critical, we have $x_{j}=a_{j+1}$, and the result follows directly from $\mathbf{P 3}$, i.e., $x_{j, B} \leq x_{j}=a_{j+1}$, and from P4, i.e., $x_{i} \leq x_{i, j}$ for all $i=1, \ldots, j$, hence, $a_{j+1}=x_{j} \leq x_{j, j}$.

Critical Intervals: According to Lemma 4.5, a critical job will occur whenever a situation arises such that $a_{j+1} \in\left[x_{j, B}, x_{j, j}\right]$ for some $j=1, \ldots, B$. To reflect this fact, we refer to the time intervals $\left[x_{j, B}, x_{j, j}\right]$ as critical intervals. Clearly, the wider the critical intervals, the greater the likelihood that the optimal solution will contain critical jobs. Once again, we remind the reader that all such critical intervals can be precomputed through Lemma 4.3, so that the condition

$$
a_{j+1} \in\left[x_{j, B}, x_{j, j}\right]
$$

is one that may be tested off line for any given arrival time $a_{1}$ and positive integer $B$.

To illustrate the use of the preceding lemmas, consider the example shown in Fig. 2 for the case $N=3$. In the figure, $x_{1,1}, x_{1,2}, x_{2,2}, x_{1,3}, x_{2,3}, x_{3,3}$ have been computed for a given arrival time $a_{1}$ and $B=1,2$, and 3. First, consider the implications of Lemma 4.4. With $\bar{N}=N=3$ and $L=1$, according to the lemma if $x_{1,2} \leq a_{2} \leq x_{1,1}$, as shown in Fig. 2(a), then job 1 is critical (regardless of $a_{3}$ ). Therefore, the optimal departure time for job 1 is $x_{1}=a_{2}$. Note that if $a_{2}<x_{1,2}$ then job 2 is definitely in the same busy period as job 1 , whereas if $a_{2}>x_{1,1}$ then job 2 must start a separate busy period. Thus, the location of $a_{2}$ relative to the critical interval $\left[x_{1,2}, x_{1,1}\right]$ allows us to determine whether job 1 is critical, whether it ends the first busy period, or whether it is included in a busy period containing at least the first two jobs. Similarly, with $L=2$, if $a_{2}<x_{1,3}$ and $x_{2,3} \leq a_{3} \leq x_{2,2}$, as shown in Fig. 2(b), then job 2 is critical.

Next, consider Lemma 4.5. Suppose that $a_{2}<x_{1,2}$ and $a_{3}<$ $x_{2,3}$. Then, with $j=2$ and $B=3$, if $x_{1,3} \leq a_{2}<x_{1,2}$ and $a_{3}<x_{2,3}$, job 1 is the only job in the busy period satisfying the condition of this lemma, and, hence, job 1 must be critical. On the other hand, suppose $x_{1,3} \leq a_{2}<x_{1,2}$ and $x_{2,3} \leq a_{3}<$ $x_{2,2}$, as shown in Fig. 2(c). In this case, both $j=1,2$ satisfy the conditions of the lemma; therefore, either or both of jobs 1 and 2 might be critical. Without explicitly solving the problem, however, it is not possible to make a final determination.

To summarize, while Lemma 4.5 can be used to determine whether or not a busy period will contain critical jobs by checking if $a_{i+1} \in\left[x_{i, B}, x_{i, i}\right]$, it cannot be used to determine which jobs in the busy period will be critical. To answer this question one must explicitly solve the problem with an iterative algorithm, unless the conditions of Lemma 4.4 are also satisfied; in that case, we can further identify the critical jobs, which significantly simplifies the effort that goes toward an explicit solution of the problem.

\section{Analysis of a Problem Class with Separable Cost STRUCTURE}

For the remainder of the paper, we concentrate on a family of problems for which the cost functions $L_{i}\left(x_{i}, u_{i}\right)$ are separable in the sense that

$$
L_{i}\left(x_{i}, u_{i}\right)=\theta_{i}\left(u_{i}\right)+\psi_{i}\left(x_{i}\right)
$$

for all $i=1, \ldots, N$. In addition, we will make the following assumptions regarding the functions $\theta_{i}(\cdot), \psi_{i}(\cdot)$, and $s_{i}(\cdot)$.

Assumption C1: For each $i=1, \ldots, N, \theta_{i}(\cdot)$ is strictly convex, twice continuously differentiable, and monotonically decreasing with $\lim _{u_{i} \rightarrow 0^{+}} \theta_{i}\left(u_{i}\right)=-\lim _{u_{i} \rightarrow 0^{+}}\left(d \theta_{i} / d u_{i}\right)=$ $\infty$ and $\lim _{u_{i} \rightarrow \infty} \theta_{i}\left(u_{i}\right)=\lim _{u_{i} \rightarrow \infty}\left(d \theta_{i} / d u_{i}\right)=0$.

Assumption C2: For each $i=1, \ldots, N, \psi_{i}(\cdot)$ is strictly convex, twice continuously differentiable, and its minimum is obtained at a finite point $\delta_{i}$. 
Assumption C3: For each $i=1, \ldots, N, s_{i}(\cdot)$ is monotonically increasing and linear: $s_{i}\left(u_{i}\right)=\alpha u_{i}, \alpha>0$.

In the context of manufacturing systems, under Assumption C3 we consider problems where processing times are proportional to the control. In the simplest case, we directly control processing times (i.e., $s_{i}=u_{i}$ ) so as to trade off quality measured through $\theta_{i}\left(u_{i}\right)$ against timely job completion measured through $\psi_{i}\left(x_{i}\right)$. For a concrete example, let $\theta_{i}\left(u_{i}\right)=1 / u_{i}$, $\psi_{i}\left(x_{i}\right)=\left(x_{i}-\delta_{i}\right)^{2}$, and $s_{i}=\alpha u_{i}$, which satisfy Assumptions C1-C3 respectively. In this case, each job is penalized for deviating from a desired target completion time $\delta_{i}$. In addition, short service times are penalized so as to ensure that each job is processed long enough to achieve its desired "quality" target [recall the stopping rule (3)]. Note that this is a different family of problems from those studied in earlier work in this framework [17], where $\theta_{i}(\cdot)$ and $\psi_{i}(\cdot)$ were strictly convex and monotonically increasing for positive arguments and processing times $s_{i}(\cdot)$ were inversely proportional to the control.

The main result of this section is to show that this class of problems possesses Properties P1-P4 identified in the previous section. Recall that it is under these properties that we were able to identify characterizations of critical jobs (Lemmas 4.4 and 4.5). This, therefore, allows us to develop iterative algorithms for the explicit solution of the problem which are computationally efficient, since they help to decompose a TPBVP into several smaller decoupled (or partially coupled) TPBVPs. The uniqueness property $\mathbf{P 1}$ is particularly interesting, because this class of optimization problems is not convex, despite conditions C1-C3; this issue is addressed in Section V-B. Note that, in order to maintain the flow of the presentation, all proofs of assertions made in this section have been placed in the Appendix.

\section{A. Generalized Gradient Properties}

Under Assumptions C1-C3, we can establish the following two properties of the generalized gradients $\xi_{i}^{-}$and $\xi_{i}^{+}$defined in (25).

Lemma 5.1: Under Assumptions C1-C3, $\xi_{i}^{-} \leq \xi_{i}^{+}$on an optimal sample path.

Proof: See the Appendix.

Lemma 5.2: Under Assumptions C1-C3, for every $\nu=i, \ldots, m(i)$ on an optimal sample path,

$$
\xi_{i}^{-}=\xi_{\nu}^{-} \quad \text { and } \quad \xi_{i}^{+}=\xi_{\nu}^{+} .
$$

Proof: See the Appendix.

Remark 5.1: The previous result can be obtained under weaker conditions than $\mathbf{C 3}$. Specifically, as long as $s_{i}\left(u_{i}\right)$ satisfy $\mathbf{A 2}$ and have bounded derivatives, the perturbations $\alpha \Delta$ and $-\alpha \Delta$ to the service times of jobs $i$ and $\nu$, used in the proof, respectively, may be replaced by $\left(d s_{i} / d u_{i}\right) \Delta$ and $-\gamma\left(d s_{i} / d u_{i}\right) \Delta$ with $\gamma=\left(d s_{\nu} / d u_{\nu}\right) /\left(d s_{i} / d u_{i}\right)$.

\section{B. Existence and Uniqueness of Optimal Control Sequence}

The existence of a nontrivial bounded solution to the optimal control problem (5) under (31) and Assumptions C1-C3 is easy to verify, and we omit it. In what follows, we establish the uniqueness of the optimal solution, a property which is not as obvious as might appear at first sight.
A sufficient, but not necessary, condition for uniqueness is the strict convexity of the objective function

$$
J\left(u_{1}, \ldots, u_{N}\right)=\sum_{i=1}^{N}\left\{\theta_{i}\left(u_{i}\right)+\psi_{i}\left(x_{i}\right)\right\}
$$

in the control sequence $\left\{u_{1}, \ldots, u_{N}\right\}$. Since the functions $\theta_{i}\left(u_{i}\right)$ are convex (from $\mathbf{C 1}$ ), their sum is convex. Thus, the convexity of $J$ depends on whether the composite functions $\psi_{i}\left(x_{i}\left(u_{1}, \ldots, u_{N}\right)\right)$ are also convex in the controls; this would be ensured if the functions $\psi_{i}(\cdot)$, in addition to being convex under C2, were also nondecreasing. However, this is not the case in our problem setting, since we only assume $\psi_{i}(\cdot)$ are strictly convex.

Example: We illustrate the nonconvexity of our cost function through the following simple example with $N=2$. Let $a_{1}=1$ and $a_{2}=2$ and define cost functions as follows:

$$
\begin{aligned}
& \theta_{1}\left(u_{1}\right)=\frac{1}{u_{1}}, \quad \theta_{2}\left(u_{2}\right)=\frac{1}{u_{2}}, \quad \psi_{1}\left(x_{1}\right)=\left(x_{1}-5\right)^{2}, \\
& \psi_{2}\left(x_{2}\right)=\left(x_{2}-10\right)^{2} .
\end{aligned}
$$

This gives the cost function

$$
\begin{aligned}
J\left(u_{1}, u_{2}\right)= & \frac{1}{u_{1}}+\frac{1}{u_{2}}+\left(x_{1}-5\right)^{2}+\left(x_{2}-10\right)^{2} \\
= & \frac{1}{u_{1}}+\frac{1}{u_{2}}+\left(a_{1}+u_{1}-5\right)^{2} \\
& +\left[\max \left(a_{1}+u_{1}, a_{2}\right)+u_{2}-10\right]^{2} \\
= & \frac{1}{u_{1}}+\frac{1}{u_{2}}+\left(1+u_{1}-5\right)^{2} \\
& +\left[\max \left(1+u_{1}, 2\right)+u_{2}-10\right]^{2} .
\end{aligned}
$$

The last term above is not a convex function of $u_{1}$, although it is convex in $u_{2}$. This nonconvexity is visualized in Fig. 3 where $J\left(u_{1}, u_{2}\right)$ is plotted. Note that there is a single optimal point for this function.

In summary, establishing the uniqueness of an optimal solution for the optimal control problem (5) under (31) and Assumptions C1-C3 is not a straightforward task. We are, nevertheless, able to prove uniqueness by proceeding in two steps. First, in Lemma 5.3, we show that the busy period structure of an optimal sample path is unique. Second, in Theorem 5.1, we show that the controls within each busy period are unique.

Lemma 5.3: Under Assumptions C1-C3, the busy period structure of an optimal sample path is unique in the sense that the indices $n(i)$, for all $i=1, \ldots, N$, are unique.

Proof: See the Appendix.

Given the uniqueness of the busy period structure, the linearity of the service functions (Assumption C3) makes it possible to establish that the controls within the busy periods are unique, and hence the entire optimal control sequence is unique.

Theorem 5.1: Under Assumptions C1-C3, the optimal control sequence is unique.

Proof: See the Appendix. 


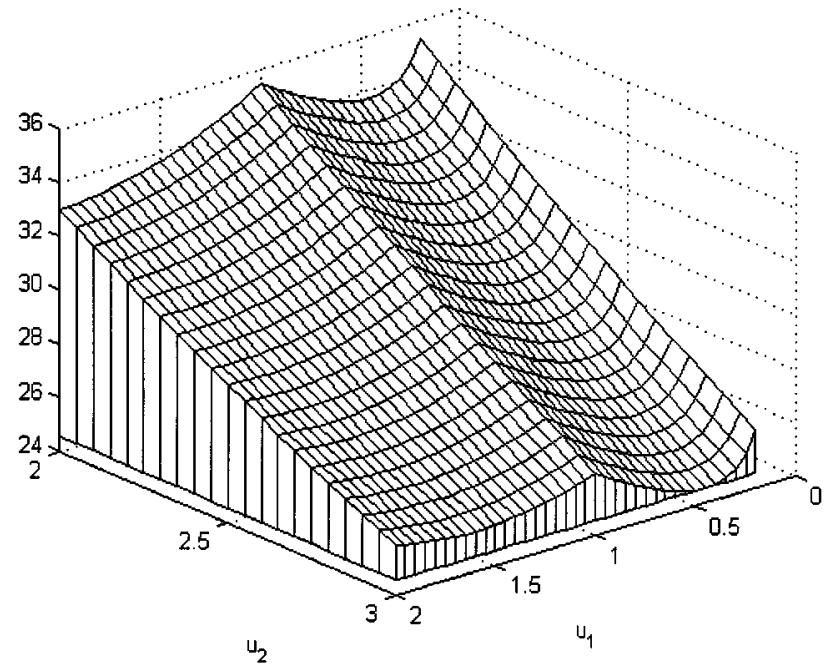

Fig. 3. An example of a nonconvex cost function $J\left(u_{1}, u_{2}\right)$.

\section{Properties of Noncritical Departures}

In Section IV-B, we presented four properties P1-P4 which allow us to derive conditions for identifying critical jobs, a crucial step for developing efficient solution algorithms for the problem. For the class of problems considered in this section, under (31) and C1-C3, we have already established the first property (uniqueness of solution) in Theorem 5.1. We shall now show that the remaining properties, P2-P4, are also satisfied.

As in previous sections, given a busy period consisting of jobs $1, \ldots, B$ on an optimal sample path, we shall denote the optimal departure times for the jobs in this busy period by $x_{1}, \ldots, x_{B}$. We also denote by $x_{i, B}$ the noncritical departure of the $i$ th job in this busy period and remind the reader that noncritical departures are quantities that may be precomputed off line for any given arrival time (initiating the busy period) and positive integer $B$. We begin by proving that Property $\mathbf{P 2}$ holds.

Theorem 5.2: Under Assumptions C1-C3 and a given arrival time $a_{1}$, the noncritical departure times are monotonically decreasing in the number of jobs in a busy period $\{1, \ldots, B\}$, i.e., $x_{i, \bar{B}} \leq x_{i, B}$ for all $i=1, \ldots, B$ and $B \leq \bar{B}$.

Proof: See the Appendix.

Note that when the noncritical departures are not monotonically decreasing in $B$ (i.e., Property $\mathbf{P 2}$ is not satisfied), then the solution may not be unique and the critical intervals discussed in the previous section shrink to points (i.e., in order for a job to be critical it must arrive exactly coincident with a noncritical departure). Thus, when the noncritical departures are not monotonically decreasing, critical jobs are not likely to occur.

In order to prove Properties $\mathbf{P 3}$ and P4, we will need the following additional result that identifies a monotonicity property of the optimal controls within a block. In particular, we show that if the end of a block is perturbed so as to increase (decrease) its length, then the optimal controls associated with all the jobs in this block must increase (decrease).

Lemma 5.4: Consider a block consisting of jobs $i=1, \ldots, L$ on an optimal sample path and let the block be such that it does not end a busy period. Under Assumptions C1-C3, $d u_{i} / d x_{L}>0$ for all $i=1, \ldots L$.

Proof: See the Appendix.

We can now prove Property P3, as shown next.

Theorem 5.3: Under Assumptions C1-C3, the noncritical departure times in a busy period lower bound the optimal departure times, i.e., $x_{i, B} \leq x_{i}$ for all $i=1, \ldots, B$.

Proof: See the Appendix.

Finally, we establish Property P4. Recall that in this case $i=1, \ldots, L$ indexes jobs within a block of $L$ jobs and $x_{i, L}$ is evaluated as a noncritical departure with respect to a busy period starting with the first job in the block and containing $L$ jobs.

Theorem 5.4: Under Assumptions C1-C3, the noncritical departure times in a block upper bound the optimal departure times, i.e., $x_{i} \leq x_{i, L}$ for all $i=1, \ldots, L$.

Proof: See the Appendix.

\section{SUMMARY AND CONCLUSION}

In this paper, we defined a hybrid system modeling framework (motivated from manufacturing environments) which combines the time-driven dynamics of various physical processes with the event-driven dynamics describing switches between the physical processes. Characteristic of the framework are "max-plus" equations describing the state dynamics. The nondifferentiability of the "max" function leads to nonsmooth optimization problems. However, exploiting properties of the optimal sample paths allows us to decompose it into a collection of independent busy periods and to partition the busy periods into blocks defined by "critical jobs." Since critical jobs are responsible for making the problem nonsmooth, we have studied their properties and derived several conditions for identifying them in an optimal sample path. For a large class of problems, we have also shown that the optimal solution is unique, despite the fact that the cost functions involved are not convex and not differentiable, and that some additional structural properties hold, which enable the development of efficient solution algorithms. The development of such algorithms is the subject of a parallel research effort. Ongoing work is aimed at extending our analysis to systems with more complex dynamics (e.g., multistage processes), incorporating uncertainty into the modeling framework, and considering problems where the control sequence is time-dependent, i.e., $u_{i}$ may vary over the duration of the physical process corresponding to the $i$ th job.

\section{APPENDIX}

Proof of Lemma 5.1: Under Assumption C3, let $d s_{i} / d u_{i}=\alpha>0$. In view of (31), Lemma 3.2 gives

$$
\xi_{i}^{+}-\xi_{i}^{-}=\alpha \sum_{j=m(i)+1}^{n(i)} \frac{d \psi_{j}}{d x_{j}}
$$

Without loss of generality, let us assume there are no critical jobs between $m(i)$ and the end of the busy period that contains 
job $i$. Then, the optimality conditions in Theorem 3.1 require that $\xi_{m(i)+1}^{-}=\xi_{m(i)+1}^{+}=0$ and we get

$$
\frac{d \theta_{m(i)+1}}{d u_{m(i)+1}}+\alpha \sum_{j=m(i)+1}^{n(i)} \frac{d \psi_{j}}{d x_{j}}=0 .
$$

By Assumption $\mathbf{C 1}, d \theta_{i} / d u_{i}<0$, from which it follows that

$$
\alpha \sum_{j=m(i)+1}^{n(i)} \frac{d \psi_{j}}{d x_{j}}>0
$$

and (33) implies that $\xi_{i}^{-} \leq \xi_{i}^{+}$.

Proof of Lemma 5.2: To show that $\xi_{i}^{-}=\xi_{\nu}^{-}$on an optimal sample path, let $i \in\{1, \ldots, N\}$ and $\nu \in\{i, \ldots, m(i)\}$. By definition (19), we have $m(\nu)=m(i)$. Moreover, for all $j \in$ $\{i, \ldots, m(i)-1\}$ we have $x_{j}>a_{j+1}$, hence

$$
x_{j}=a_{i}+\sum_{k=i}^{j} s_{k} \quad \text { for all } j=i, \ldots, m(i) .
$$

Consider a perturbation $\Delta$ in $u_{i}$ about its optimal value and a simultaneous perturbation $-\Delta$ in $u_{\nu}$. Under $\mathbf{C 3}$, let $d s_{i} / d u_{i}=$ $\alpha>0$. It follows that the perturbed service times of jobs $i$ and $\nu$ are $s_{i}+\alpha \Delta$ and $s_{\nu}-\alpha \Delta$ respectively. For $\Delta$ sufficiently close to 0 , we can preserve the inequality $x_{j}>a_{j+1}$ for all $j \in\{i, \ldots, m(i)-1\}$ and leave $x_{m(i)}$ unaffected. Consequently, $\bar{J}(\cdot)$ is locally continuously differentiable in $\Delta$ about $\Delta=0$. In addition, since we are assuming an optimal sample path, $d \bar{J}(\Delta) / d \Delta=0$ at $\Delta=0$.

Clearly, the only effects of $\Delta$ on $\bar{J}(\cdot)$ come from the terms $\theta_{i}\left(u_{i}+\Delta\right), \theta_{\nu}\left(u_{\nu}-\Delta\right)$, and $\psi_{j}\left(x_{j}+\alpha \Delta\right)$ for $j=i, \ldots, \nu-1$, since the departure times of jobs $i, \ldots, \nu-1$ are perturbed as a result of $\Delta$ through (34). Therefore,

$$
\left.\frac{d \bar{J}(\Delta)}{d \Delta}\right|_{\Delta=0}=\frac{d \theta_{i}}{d u_{i}}+\alpha \sum_{j=i}^{\nu-1} \frac{d \psi_{j}}{d x_{j}}-\frac{d \theta_{\nu}}{d u_{\nu}}=0 .
$$

Adding and subtracting the term $\alpha \sum_{j=\nu}^{m(i)}\left(d \psi_{j} / d x_{j}\right)$ above gives

$\frac{d \theta_{i}}{d u_{i}}+\alpha \sum_{j=i}^{m(i)} \frac{d \psi_{j}}{d x_{j}}-\left(\frac{d \theta_{\nu}}{d u_{\nu}}+\alpha \sum_{j=\nu}^{m(i)} \frac{d \psi_{j}}{d x_{j}}\right)=\xi_{i}^{-}-\xi_{\nu}^{-}=0$

where we have used the definition (23) and the fact that $m(i)=$ $m(\nu)$. This establishes the first part of (32). The second part follows directly from Lemma 3.2.

Proof of Lemma 5.3: The proof is by contradiction. In particular, suppose that, for a given arrival sequence, there exist two different sample paths that both satisfy the optimality conditions in Theorem 3.1; we shall then establish a contradiction.

Due to the idle period decoupling property (Lemma 4.1), we can assume, without loss of generality, that the difference between the two sample paths is in their respective first busy pe- riods. Let us denote the two sample paths by $A$ and $B$, respectively. Let $a$ be the last job in the first busy period on sample path $A, b$ be the last job in the first busy period on sample path $B$, and assume (without loss of generality) that $a<b$. Using the subscripts $A$ and $B$ to indicate variables on the corresponding sample paths, we will show that, for all $i=1, \ldots, a$, the following two inequalities hold

$$
x_{i, A}<x_{i, B}
$$

and,

$$
s_{i, A}>s_{i, B}
$$

In view of the state equations $x_{i, A}=a_{1}+\sum_{j=1}^{i} s_{i, A}$ and $x_{i, B}=a_{1}+\sum_{j=1}^{i} s_{i, B}$, these two inequalities clearly contradict one another. This contradiction implies that $a=b$, i.e., the busy periods must coincide, and the proof is complete. Thus, it remains to prove that (35) and (36) indeed hold under the assumption $a<b$.

We prove (35) and (36) through a backward induction argument. That is, we first show the result for job $a$, then assume the result holds for jobs $i=k, \ldots, a(1<k<a)$, and prove that it must also hold for job $i=k-1$.

For job $a$, we proceed as follows. On sample path $A$, job $a$ ends the first busy period, in which case we must have $x_{a, A}<a_{a+1}$. On sample path $B$, however, job $a$ does not end the first busy period, implying that $x_{a, B} \geq a_{a+1}$. Consequently, $x_{a, A}<x_{a, B}$, establishing (35) for $i=a$.

To establish (36) for job $a$, first note that since on sample path $A$ job $a$ is the last job in the first busy period, Theorem 3.1 requires that $\xi_{a, A}^{-}=\xi_{a, A}^{+}=0$. In view of (23) and (31) this implies that

$$
\frac{d \theta_{a}}{d u_{a, A}}+\alpha \frac{d \psi_{a}}{d x_{a, A}}=0 .
$$

On sample path $B$, however, jobs $a$ and $a+1$ are in the same busy period. If job $a$ is critical, then Theorem 3.1 requires that $\xi_{a, B}^{-}$and $\xi_{a, B}^{+}$have opposite sign, which in view of Lemma 5.1 implies that

$$
\xi_{a, B}^{-}=\frac{d \theta_{a}}{d u_{a, B}}+\alpha \frac{d \psi_{a}}{d x_{a, B}}<0 .
$$

If, on the other hand, job $a$ is not critical, then $m_{B}(a)=m_{B}(a+$ 1) $>a$ and by Lemma 5.2, we have $\xi_{a, B}^{-}=\xi_{a+1, B}^{-}$. Thus, from (23) and (31),

$$
\begin{aligned}
\xi_{a, B}^{-}= & \frac{d \theta_{a}}{d u_{a, B}}+\alpha \sum_{j=a}^{m_{B}(a)} \frac{d \psi_{j}}{d x_{j, B}}=\frac{d \theta_{a+1}}{d u_{a+1, B}} \\
& +\alpha \sum_{j=a+1}^{m_{B}(a)} \frac{d \psi_{j}}{d x_{j, B}}=\xi_{a+1, B}^{-} .
\end{aligned}
$$

By subtracting common terms above, we obtain

$$
\frac{d \theta_{a}}{d u_{a, B}}+\alpha \frac{d \psi_{a}}{d x_{a, B}}=\frac{d \theta_{a+1}}{d u_{a+1, B}}<0
$$


where the inequality follows from $\mathbf{C 1}$. We have, therefore, established that

$$
\frac{d \theta_{a}}{d u_{a, B}}+\alpha \frac{d \psi_{a}}{d x_{a, B}}<0
$$

regardless of whether job $a$ is critical or not. Since we have already established that $x_{a, A}<x_{a, B}$, we have, by C2, $d \psi_{a} / d x_{a, A}<d \psi_{a} / d x_{a, B}$. Therefore, comparing (37) and (38) it follows that:

$$
\frac{d \theta_{a}}{d u_{a, A}}>\frac{d \theta_{a}}{d u_{a, B}}
$$

which, by C1, implies that $u_{a, A}>u_{a, B}$. Finally, because of C3, this establishes (36) for job $a$.

Next, suppose that (35) and (36) are both satisfied for jobs $i=k, \ldots, a(1<k<a)$. We will now proceed to show that they are also satisfied for $i=k-1$.

First, from the state equation (14) we have $x_{k, A}=x_{k-1, A}+$ $s_{k, A}$ and $x_{k, B}=x_{k-1, B}+s_{k, B}$. Since (35) and (36) hold for job $k$, it immediately follows that $x_{k-1, A}<x_{k-1, B}$, i.e., (35) is satisfied for job $k-1$.

Now, consider (36). On sample path $A$, if job $k-1$ is not critical and there are no critical jobs between job $k-1$ and job $a$ (which ends the busy period on sample path $A$ ), then Theorem 3.1 requires that $\xi_{k-1, A}^{+}=0$. If, on the other hand, job $k-1$ is critical or there are critical jobs between job $k-1$ and job $a$, then Theorem 3.1 and Lemma 5.1 require that $\xi_{k-1, A}^{+}>0$. In view of (24) and (31), we have, therefore, established that

$$
\xi_{k-1, A}^{+}=\frac{d \theta_{k-1}}{d u_{k-1, A}}+\alpha \sum_{j=k-1}^{a} \frac{d \psi_{j}}{d x_{j, A}} \geq 0 .
$$

Next, for all $i=k-1, \ldots, a$, we have $x_{i, A} \geq a_{i+1}$. Since, as shown above, (35) holds for all $i=k-1, \ldots, a$, we have $x_{i, A}<x_{i, B}, i=k-1, \ldots, a$, and it follows that $x_{i, B}>a_{i+1}$ for all $i=k-1, \ldots, a$. This means that on sample path $B$ there can be no critical jobs between $k-1$ and $a$. Regarding job $a$, there are two cases. First, if $a$ is critical, i.e., $m_{B}(k-1)=a$, then by Theorem 3.1 and Lemma 5.1,

$$
\xi_{k-1, B}^{-}=\frac{d \theta_{k-1}}{d u_{k-1, B}}+\alpha \sum_{j=k-1}^{a} \frac{d \psi_{j}}{d x_{j, B}}<0 .
$$

Second, if job $a$ is not critical, we have $m_{B}(k-1)=m_{B}(a+1)$ and, by Lemma $5.2, \xi_{k-1, B}^{-}=\xi_{a+1, B}^{-}$. Thus, from (23)

$\frac{d \theta_{k-1}}{d u_{k-1, B}}+\alpha \sum_{j=k-1}^{m_{B}(k-1)} \frac{d \psi_{j}}{d x_{j, B}}=\frac{d \theta_{a+1}}{d u_{a+1, B}}+\alpha \sum_{j=a+1}^{m_{B}(k-1)} \frac{d \psi_{j}}{d x_{j, B}}$

which after subtracting common terms gives

$$
\frac{d \theta_{k-1}}{d u_{k-1, B}}+\alpha \sum_{j=k-1}^{a} \frac{d \psi_{j}}{d x_{j, B}}=\frac{d \theta_{a+1}}{d u_{a+1, B}}<0
$$

where the inequality follows from $\mathbf{C 1}$. This proves that

$$
\frac{d \theta_{k-1}}{d u_{k-1, B}}+\alpha \sum_{j=k-1}^{a} \frac{d \psi_{j}}{d x_{j, B}}<0
$$

whether job $a$ is critical or not. Since, as already shown above, (35) holds for all $k-1, \ldots, a$, it follows by Assumption C2 (strict convexity) that

$$
\sum_{j=k-1}^{a} \frac{d \psi_{j}}{d x_{j, A}}<\sum_{j=k-1}^{a} \frac{d \psi_{j}}{d x_{j, B}}
$$

Therefore, comparing (39) and (40) it follows that

$$
\frac{d \theta_{k-1}}{d u_{k-1, A}}>\frac{d \theta_{k-1}}{d u_{k-1, B}}
$$

and the strict convexity of $\theta_{k-1}(\cdot)$ in $\mathbf{C 1}$ implies that $u_{k-1, A}>$ $u_{k-1, B}$. By Assumption C3, this yields (36) for job $k-1$ and completes the inductive argument, thus establishing (35) and (36) which were needed to complete the proof.

Proof of Theorem 5.1: Consider a busy period on the optimal sample path that consists of jobs $\{k, \ldots, n\}$. From Lemma 5.3, the order of this busy period in the sample path and its composition $\{k, \ldots, n\}$ are unique. By Assumption C3, let $s_{i}\left(u_{i}\right)=\alpha u_{i}+\beta$ with $\alpha>0$. Thus, the optimal control sequence $\left\{u_{k}, \ldots, u_{n}\right\}$ minimizes the cost function

$$
\begin{aligned}
J_{k, n}\left(u_{k}, \ldots, u_{n}\right) \\
=\sum_{i=k}^{n}\left\{\theta_{i}\left(u_{i}\right)+\psi_{i}\left(x_{i}\right)\right\} \\
=\sum_{i=k}^{n}\left\{\theta_{i}\left(u_{i}\right)+\psi_{i}\left(a_{k}+\sum_{j=k}^{i}\left(\alpha u_{j}+\beta\right)\right)\right\}
\end{aligned}
$$

subject to the linear constraints

$$
\begin{aligned}
a_{k}+\sum_{j=k}^{i}\left(\alpha u_{j}+\beta\right) & \geq a_{i}, \quad i=k, \ldots, n-1 \\
a_{k}+\sum_{j=k}^{n}\left(\alpha u_{j}+\beta\right) & <a_{n+1} \\
u_{i} & \geq 0, \quad i=k, \ldots, n .
\end{aligned}
$$

Using the strict convexity of $\theta_{i}(\cdot)$ in Assumption C1, the function $\sum_{i=k}^{n} \theta_{i}\left(u_{i}\right)$ is strictly convex as the sum of strictly convex functions. Using the strict convexity of $\psi_{i}(\cdot)$ in Assumption C2, the function $\psi_{i}\left(a_{k}+\sum_{j=k}^{i}\left(\alpha u_{j}+\beta\right)\right)$ is convex, as a strictly convex function of a linear function in $u_{k}, \ldots, u_{i}$ (note, however,that it is not necessarily strictly convex). Therefore, $J_{k, n}\left(u_{k}, \ldots, u_{n}\right)$ is the sum of the strictly convex function $\sum_{i=k}^{n} \theta_{i}\left(u_{i}\right)$ and the convex function $\sum_{i=k}^{n} \psi_{i}\left(a_{k}+\sum_{j=k}^{i}\left(\alpha u_{j}+\beta\right)\right)$, which yields a strictly convex function. Thus, the problem of minimizing (41) subject to the constraints (42) is a convex program, which, therefore, 
has a unique solution. Since there is a unique optimal control sequence for jobs in every busy period and since the busy period structure itself is unique from Lemma 5.3, it follows that the optimal control sequence is unique.

Proof of Theorem 5.2: Consider a busy period containing $B$ jobs on an optimal sample path, and suppose that none of the jobs in the busy period are critical. By idle period decoupling (Lemma 4.1), we lose no generality by indexing the first job in this busy period as $i=1$, in which case the last job has index $B$. Now, suppose we change the arrival sequence in such a way that when the optimal controls are recomputed, the busy period now contains $\bar{B}>B$ jobs, none of which are critical. We now proceed to show that the optimal departure times (coinciding with the noncritical departure times since the busy periods do not contain any critical jobs) satisfy $x_{i, \bar{B}}<x_{i, B}$ for all $i=$ $1, \ldots, B$.

The proof is by induction. We begin by showing the result for job $i=1$ (basis step). Then, assuming the result holds for jobs $i=1, \ldots, k<B-1$, we show that it also holds for job $i=k+1$.

The proof of $x_{1, \bar{B}}<x_{1, B}$ is by contradiction. Suppose that $x_{1, \bar{B}} \geq x_{1, B}$. Then, since both busy periods begin at a common time, the state equation (14) and $\mathbf{C 3}$ imply that $u_{1, \bar{B}} \geq u_{1, B}$. As a consequence, Assumptions $\mathbf{C} 1$ and $\mathbf{C 2}$ give $d \theta_{1} / d u_{1}, \bar{B} \geq$ $d \theta_{1} / d u_{1, B}$ and $d \psi_{1} / d x_{1, \bar{B}} \geq d \psi_{1} / d x_{1, B}$. Recalling Theorem 3.1 , optimality of the controls $u_{1}, \bar{B}$ and $u_{1, B}$ requires that

$$
\frac{d \theta_{1}}{d u_{1, \bar{B}}}+\alpha \sum_{j=1}^{\bar{B}} \frac{d \psi_{j}}{d x_{j, \bar{B}}}=\frac{d \theta_{1}}{d u_{1, B}}+\alpha \sum_{j=1}^{B} \frac{d \psi_{j}}{d x_{j, B}}=0
$$

which, in light of the two previous inequalities, implies that

$$
\alpha \sum_{j=2}^{\bar{B}} \frac{d \psi_{j}}{d x_{j, \bar{B}}} \leq \alpha \sum_{j=2}^{B} \frac{d \psi_{j}}{d x_{j, B}} .
$$

Continuing, optimality of the controls $u_{2, \bar{B}}$ and $u_{2, B}$ requires that

$$
\frac{d \theta_{2}}{d u_{2, \bar{B}}}+\alpha \sum_{j=2}^{\bar{B}} \frac{d \psi_{j}}{d x_{j, \bar{B}}}=\frac{d \theta_{2}}{d u_{2, B}}+\alpha \sum_{j=2}^{B} \frac{d \psi_{j}}{d x_{j, B}}=0
$$

which, given (43), implies that $d \theta_{2} / d u_{2, \bar{B}} \geq d \theta_{2} / d u_{2, B}$. By Assumption C1, it follows that $u_{2, \bar{B}} \geq u_{2, B}$. Substituting this into the state equation, and recalling the assumption $x_{1}, \bar{B} \geq$ $x_{1, B}$, we get $x_{2, \bar{B}} \geq x_{2, B}$. Thus, by Assumption C2, we have, $d \psi_{2} / d x_{2, \bar{B}} \geq d \psi_{2} / d x_{2, B}$, which, from (44), gives

$$
\alpha \sum_{j=3}^{\bar{B}} \frac{d \psi_{j}}{d x_{j, \bar{B}}} \leq \alpha \sum_{j=3}^{B} \frac{d \psi_{j}}{d x_{j, B}} .
$$

This argument is carried forward for jobs $i=3, \ldots, B$ leading to the conclusion

$$
\alpha \sum_{j=B+1}^{\bar{B}} \frac{d \psi_{j}}{d x_{j, \bar{B}}} \leq 0 .
$$

This, however, is a contradiction, since optimality for job $B+1$ requires

$$
\frac{d \theta_{B+1}}{d u_{B+1, \bar{B}}}+\alpha \sum_{j=B+1}^{\bar{B}} \frac{d \psi_{j}}{d x_{j, \bar{B}}}=0
$$

which, given $\mathbf{C 1}$, requires

$$
\alpha \sum_{j=B+1}^{\bar{B}} \frac{d \psi_{j}}{d x_{j, \bar{B}}}>0 .
$$

In summary, assuming $x_{1, \bar{B}} \geq x_{1, B}$ leads to a contradiction and this establishes the inequality $x_{1, \bar{B}}<x_{1, B}$.

Next, assuming the result holds for jobs $i=1, \ldots, k<$ $B-1$ we will show that it holds for $i=k+1$. The proof here is virtually identical to the one used above for job $i=1$. That is, suppose that $x_{k+1, \bar{B}} \geq x_{k+1, B}$. The state equation gives

$$
x_{k+1, \bar{B}}=x_{k, \bar{B}}+\alpha u_{k+1, \bar{B}} \geq x_{k, B}+\alpha u_{k+1, B}=x_{k+1, B}
$$

and, since the result holds for all $i=1, \ldots, k$, we must have $u_{k+1, \bar{B}} \geq u_{k+1, B}$. Thus, by $\mathbf{C 1}, d \theta_{k+1} / d u_{k+1, \bar{B}}$ $\geq \quad d \theta_{k+1} / d u_{k+1, B}$, and, by $\mathbf{C 2}, d \psi_{k+1} / d x_{k+1, \bar{B}}$ $\geq d \psi_{k+1} / d x_{k+1, B}$. Using these inequalities and the optimality equation

$\frac{d \theta_{k+1}}{d u_{k+1, \bar{B}}}+\alpha \sum_{j=k+1}^{\bar{B}} \frac{d \psi_{j}}{d x_{j, \bar{B}}}=\frac{d \theta_{k+1}}{d u_{k+1, B}}+\alpha \sum_{j=k+1}^{B} \frac{d \psi_{j}}{d x_{j, B}}=0$

we infer that

$$
\alpha \sum_{j=k+2}^{\bar{B}} \frac{d \psi_{j}}{d x_{j, \bar{B}}} \leq \alpha \sum_{j=k+2}^{B} \frac{d \psi_{j}}{d x_{j, B}} .
$$

Proceeding exactly as before, we arrive at the conclusion that

$$
\alpha \sum_{j=B+1}^{\bar{B}} \frac{d \psi_{j}}{d x_{j, \bar{B}}} \leq 0
$$

which, by the optimality of the control for job $B+1$ in (46) and $\mathbf{C 1}$, gives a contradiction. This contradiction establishes that $x_{k+1, \bar{B}}<x_{k+1, B}$, and, hence, completes the proof.

Proof of Lemma 5.4: Consider the conditions that must be satisfied by the jobs in a block on an optimal sample path. These conditions are obtained from the cost function

$$
J=\sum_{i=1}^{L}\left\{\theta_{i}\left(u_{i}\right)+\psi_{i}\left(x_{i}\right)\right\}
$$

subject to

$$
x_{i}=a_{1}+\alpha \sum_{j=1}^{i} u_{j} \quad \text { and } \quad x_{L}=a_{L+1}
$$

as long as the block is not the last in a busy period. Adjoining the two constraints to the cost gives

$$
\begin{aligned}
\bar{J}= & \sum_{i=1}^{L}\left\{\theta_{i}\left(u_{i}\right)+\psi_{i}\left(x_{i}\right)+\lambda_{i}\left[a_{1}+\alpha \sum_{j=1}^{i} u_{j}-x_{i}\right]\right\} \\
& +\nu\left(x_{L}-a_{L+1}\right)
\end{aligned}
$$


where $\lambda_{i}$ is the $i$ th costate and $\nu$ is an additional multiplier. The necessary conditions for optimality that must be satisfied by the jobs in the block are

$$
0=\frac{d \theta_{i}}{d u_{i}}+\alpha \sum_{j=i}^{L} \lambda_{j}, \quad \lambda_{i}=\frac{d \psi_{i}}{d x_{i}}
$$

for all $i=1, \ldots, L$, along with boundary conditions $x_{L}=$ $a_{L+1}$ and $\lambda_{L}=d \psi_{L} / d x_{L}$. Comparing the optimality equations for $i=1,2$, we get

$$
\frac{d \theta_{1}}{d u_{1}}+\alpha \sum_{j=1}^{L} \frac{d \psi_{j}}{d x_{j}}=\frac{d \theta_{2}}{d u_{2}}+\alpha \sum_{j=2}^{L} \frac{d \psi_{j}}{d x_{j}}=0 .
$$

Cancelling common terms we get

$$
\frac{d \theta_{1}}{d u_{1}}+\alpha \frac{d \psi_{1}}{d x_{1}}=\frac{d \theta_{2}}{d u_{2}}
$$

and differentiating with respect to $x_{L}$ gives

$$
\frac{d^{2} \theta_{1}}{d u_{1}^{2}} \frac{d u_{1}}{d x_{L}}+\alpha \frac{d^{2} \psi_{1}}{d x_{1}^{2}} \frac{d x_{1}}{d x_{L}}=\frac{d^{2} \theta_{2}}{d u_{2}^{2}} \frac{d u_{2}}{d x_{L}} .
$$

Recalling that $x_{1}=a_{1}+\alpha u_{1}$, therefore, $d x_{1} / d x_{L}=$ $\alpha\left(d u_{1} / d x_{L}\right)$, we get

$$
\left(\frac{d^{2} \theta_{1}}{d u_{1}^{2}}+\alpha^{2} \frac{d^{2} \psi_{1}}{d x_{1}^{2}}\right) \frac{d u_{1}}{d x_{L}}=\frac{d^{2} \theta_{2}}{d u_{2}^{2}} \frac{d u_{2}}{d x_{L}} .
$$

By the strict convexity of the functions $\theta$ and $\psi$ (Assumptions C1 and C2), the expression above implies that $d u_{1} / d x_{L}$ and $d u_{2} / d x_{L}$ must have the same sign.

Proceeding the same way for $i=2,3$, we can show that $d u_{1} / d x_{L}, d u_{2} / d x_{L}$, and $d u_{3} / d x_{L}$ all have the same sign. Continuing the argument for all remaining $i=4, \ldots, L$, we reach the conclusion that all $d u_{i} / d x_{L}, i=1, \ldots, L$, have the same sign. Moreover, note that

$$
x_{L}=a_{1}+\alpha \sum_{j=1}^{L} u_{j} .
$$

and differentiating with respect to $x_{L}$ gives

$$
1=\alpha \sum_{j=1}^{L} \frac{d u_{j}}{d x_{L}}
$$

implying that $d u_{j} / d x_{L}>0$ for at least one $j \in\{1, \ldots, L\}$. However, since all $d u_{i} / d x_{L}, i=1, \ldots, L$, have the same sign, it follows that $d u_{i} / d x_{L}>0$ for all $i=1, \ldots, L$.

Proof of Theorem 5.3: Consider a busy period consisting of $B$ jobs on an optimal sample path. As usual, there is no loss of generality if we index the first job in this busy period as $i=1$, in which case the last job in the busy period is $i=B$. Denoting the noncritical departure times by $x_{1, B}, \ldots, x_{B, B}$ and the optimal departures by $x_{1}, \ldots, x_{B}$, we will now show that, for a fixed $a_{1}, x_{i, B} \leq x_{i}$ for all $i=1, \ldots, B$.

The result is trivial when the busy period does not contain any critical jobs, since in this case the optimal departures coincide, by definition, with the noncritical departures, i.e., $x_{i, B}=x_{i}$ for $i=1, \ldots, B$. Let us, therefore, consider a busy period that contains at least one critical job. Consider the inequality for the case $i=B$, and suppose that it does not hold, i.e., let $x_{B, B}>x_{B}$. By Assumption C2, this implies that $d \psi_{B} / d x_{B, B}$ $>d \psi_{B} / d x_{B}$. Recalling the optimality equation from Theorem 3.1 for job $B$ in this case, the noncritical departures and corresponding controls must satisfy

$$
\xi_{B, B}^{+}=\frac{d \theta_{B}}{d u_{B, B}}+\alpha \frac{d \psi_{B}}{d x_{B, B}}=0
$$

whereas in the busy period that contains at least one critical job we have

$$
\xi_{B}^{+}=\frac{d \theta_{B}}{d u_{B}}+\alpha \frac{d \psi_{B}}{d x_{B}}=0
$$

since job $B$ cannot be the critical one. Comparing the last two equalities and in view of $d \psi_{B} / d x_{B, B}>d \psi_{B} / d x_{B}$, we must have $d \theta_{B} / d u_{B, B}<d \theta_{B} / d u_{B}$, which, by Assumption $\mathbf{C 1}$, gives $u_{B, B}<u_{B}$.

Using the state equation and $\mathbf{C 3}$, we have

$$
x_{B, B}=x_{B-1, B}+\alpha u_{B, B}>x_{B-1}+\alpha u_{B}=x_{B}
$$

and it follows that $x_{B-1, B}>x_{B-1}$.

Next, consider the optimality equation for job $B-1$. The noncritical departures and corresponding controls must satisfy

$$
\xi_{B-1, B}^{+}=\frac{d \theta_{B-1}}{d u_{B-1, B}}+\alpha \sum_{j=B-1}^{B} \frac{d \psi_{j}}{d x_{j, B}}=0
$$

whereas in the busy period that contains at least one critical job we have

$$
\xi_{B-1}^{+}=\frac{d \theta_{B-1}}{d u_{B-1}}+\alpha \sum_{j=B-1}^{B} \frac{d \psi_{j}}{d x_{j}} \geq 0
$$

where the inequality accounts for the fact that it is possible that job $B-1$ is critical. Since we have shown that $x_{i, B}>x_{i}$ for $i=B-1, B$, it follows from $\mathbf{C} 2$ that $d \psi_{i} / d x_{i, B}>d \psi_{i} / d x_{i}$ for $i=B-1, B$. Therefore, comparing the two equations above, we conclude that $d \theta_{B-1} / d u_{B-1, B}<d \theta_{B-1} / d u_{B-1}$, which by C1, gives $u_{B-1, B}<u_{B-1}$.

Continuing this argument for jobs $i=B-2, \ldots, 1$, we arrive at the conclusion that $x_{1, B}>x_{1}$ and $u_{1, B}<u_{1}$. However

$$
x_{1, B}=a_{1}+\alpha u_{1, B}>x_{1}=a_{1}+\alpha u_{1}
$$

implying that $u_{1, B}>u_{1}$ which contradicts $u_{1, B}<u_{1}$. This contradiction establishes the result for job $i=B$.

We can now use the inequality $x_{B, B} \leq x_{B}$, just established, to show the result for all of the other jobs in the last block in the busy period. Thus, if $j$ is the last critical job in the busy period, we have $\xi_{i}^{+}=0$ for all $i=j+1, \ldots, B-1$. For job $B$, using the optimality equation as before, we have

$$
\frac{d \theta_{B}}{d u_{B, B}}+\alpha \frac{d \psi_{B}}{d x_{B, B}}=\frac{d \theta_{B}}{d u_{B}}+\alpha \frac{d \psi_{B}}{d x_{B}}=0 .
$$

Since $x_{B, B} \leq x_{B}$ we have (by C2) $d \psi_{B} / d x_{B, B} \leq d \psi_{B} / d x_{B}$, therefore the equation above implies that $d \theta_{B} / d u_{B}, B$ $\geq d \theta_{B} / d u_{B}$. By $\mathbf{C 1}$, this implies that $u_{B, B} \geq u_{B}$. Using the state equation and $\mathbf{C} \mathbf{3}$, we then get

$$
x_{B-1, B}=x_{B, B}-\alpha u_{B, B} \leq x_{B}-\alpha u_{B}=x_{B-1} .
$$

Repeating this process for $i=j+1, \ldots, B-1$, we establish the result for every job in the last block, including the last critical job in the busy period. 
It remains to show that the result holds for the remaining jobs in the busy period. To do so, let us first suppose that the busy period contains only two blocks, i.e., only one critical job. Index the first job in the busy period as 1 , the critical job as $L$, and the last job as $B$. We just showed that $x_{i, B} \leq x_{i}$ for all $i=L, \ldots, B$, hence $x_{L, B} \leq x_{L}$. Recalling Lemma 4.2, the optimal controls, and hence optimal departures, in a block of jobs $\{1, \ldots, L\}$ are obtained as the solution of an optimization problem involving the cost functions $L_{1}\left(x_{1}, u_{1}\right), \ldots, L_{L}\left(x_{L}, u_{L}\right)$ satisfying $\mathbf{A 1}$, with the terminal constraint $x_{L}=a_{L+1}$. Thus, for all $i=1, \ldots, L$, $u_{i}\left(a_{L+1}\right)$ and $x_{i}\left(a_{L+1}\right)$ are continuous functions of $a_{L+1}$ for $a_{L+1} \leq x_{L, L}$. When $a_{L+1}=x_{L, L}$ we get $x_{i}\left(a_{L+1}\right)=x_{i, L}$ and for all $a_{L+1}>x_{L, L}$ we have $x_{i}\left(a_{L+1}\right)=x_{i, L}$ since the block becomes a busy period without any critical jobs, therefore the optimal departures are given by the noncritical departures $x_{1, L}, \ldots, x_{L, L}$. We may now use Lemma 5.4 , which asserts that $d u_{i} / d x_{L}>0$, therefore, (from the state equation and $\mathbf{C 3}$ ) $d x_{i} / d x_{L}>0$ for all $i=1, \ldots, L$ in the block. In particular, since $x_{L, B} \leq x_{L}$

$$
x_{L, B}=a_{1}+\alpha \sum_{j=1}^{L} u_{j, B} \leq a_{1}+\alpha \sum_{j=1}^{L} u_{j}=x_{L}
$$

i.e., the sum of the optimal controls in the block is greater than the sum of the controls under noncritical departures. Thus, at least one of the controls must have increased as the length of the block increases from $x_{L, B}$ to $x_{L}$. From Lemma 5.4, however, this immediately implies that the controls for all jobs must have increased. Hence, the departure times of all the jobs in the block increase, thus establishing the inequality $x_{i, B} \leq x_{i}$ for all $i=$ $1, \ldots, L-1$.

Finally, we must show the result also holds when the busy period has more than one block, i.e., two or more critical jobs. Let $k, j$ be the first and second critical job respectively in a busy period with three blocks. Then, consider jobs $\{k+1, \ldots, B\}$ and note that the noncritical departures $x_{k+1, B-k}, \ldots, x_{B-k, B-k}$ depend only on $a_{k}$ and on $B-k$ (Lemma 4.3). Therefore, we may treat $\{k+1, \ldots, B\}$ as a separate busy period initiated by $a_{k+1}$ for the purpose of evaluating these noncritical departures. Moreover, $x_{k+1}, \ldots, x_{j}$ depend only on $a_{k+1}$ and $a_{j+1}$ (Lemma 4.2) and, similarly for $x_{j+1}, \ldots, x_{B}$, i.e., $x_{k+1}, \ldots, x_{B}$ are independent of any arrival times prior to $a_{k+1}$. Therefore, the result previously obtained for two blocks over $\{1, \ldots, B\}$, applies to the two blocks $\{k+1, \ldots, j\}$ and $\{j+1, \ldots, B\}$, and by repeating this argument to more than three blocks the proof is complete.

Proof of Theorem 5.4: Consider a block consisting of $L$ jobs on an optimal sample path. Index the first job in the block as job 1 and suppose the block begins at time $a_{1}$. We begin by showing the result for job $L$, i.e., $x_{L} \leq x_{L}, L$. Because job $L$ is critical, the optimality condition in Theorem 3.1 gives

$$
\xi_{L}^{-}=\frac{d \theta_{L}}{d u_{L}}+\alpha \frac{d \psi_{L}}{d x_{L}} \leq 0 .
$$

By the definition of $x_{L, L}$, it must satisfy

$$
\frac{d \theta_{L}}{d u_{L, L}}+\alpha \frac{d \psi_{L}}{d x_{L, L}}=0
$$

Comparing the two equations above we get

$$
\frac{d \theta_{L}}{d u_{L}}+\alpha \frac{d \psi_{L}}{d x_{L}} \leq \frac{d \theta_{L}}{d u_{L, L}}+\alpha \frac{d \psi_{L}}{d x_{L, L}} .
$$

Now, assume that $x_{L}>x_{L, L}$. If this is true, then $d \psi_{L} / d x_{L}$ $>d \psi_{L} / d x_{L, L}$ (Assumption C2). The inequality above then implies that $d \theta_{L} / d u_{L}<d \theta_{L} / d u_{L . L}$, which, by $\mathbf{C} 1$, implies $u_{L}<u_{L}, L$. Invoking the state equation and $\mathbf{C 3}$, we get

$$
x_{L-1}=x_{L}-\alpha u_{L}>x_{L, L}-\alpha u_{L, L}=x_{L-1, L}
$$

and it follows that $d \psi_{L-1} / d x_{L-1}>d \psi_{L-1} / d x_{L-1, L}$. Repeating the process for job $L-1$ (which is not critical) we get from Theorem 3.1

$$
\frac{d \theta_{L-1}}{d u_{L-1}}+\alpha \sum_{j=L-1}^{L} \frac{d \psi_{j}}{d x_{j}}=0
$$

and, by the definition of $x_{L-1, L}$

$$
\frac{d \theta_{L-1}}{d u_{L-1, L}}+\alpha \sum_{j=L-1}^{L} \frac{d \psi_{j}}{d x_{j, L}}=0 .
$$

Comparing the two equations in view of the inequalities previously derived, i.e., $d \psi_{i} / d x_{i}>d \psi_{i} / d x_{i, L}$ for $i=L-1, L$, we conclude that $d \theta_{L-1} / d u_{L-1}<d \theta_{L-1} / d u_{L-1, L}$, therefore (by C1) $u_{L-1}<u_{L-1, L}$. Using the state equation and $\mathbf{C 3}$ as before, we get $x_{L-2}>x_{L-2, L}$. Continuing this argument for jobs $i=L-3, \ldots, 1$ we finally get $u_{1}<u_{1, L}, x_{1}>x_{1, L}$. The state equation and $\mathbf{C 3}$ once again give

$$
x_{1}=a_{1}+\alpha u_{1}>a_{1}+\alpha u_{1, L}=x_{1, L}
$$

which contradicts the fact that $u_{1}<u_{1}, L$. This contradiction establishes that $x_{L} \leq x_{L, L}$.

Given $x_{L} \leq x_{L, L}$, the result for the remainder of the jobs in the block follows from Lemma 5.4, again noting (as in the proof of the previous lemma) that $u_{i}\left(a_{L+1}\right)$ is a continuous function of $a_{L+1}=x_{L}$. In particular, note that

$$
x_{L}=a_{1}+\alpha \sum_{j=1}^{L} u_{j} \leq a_{1}+\alpha \sum_{j=1}^{L} u_{j, L}=x_{L, L}
$$

i.e., the sum of the controls in the block decreases relative to the controls under noncritical departures. Thus, as the length of the block decreases from $x_{L, L}$ to $x_{L}$ at least one of the controls must decrease. From Lemma 5.4, however, this immediately implies that the controls for all jobs must have decreased. Hence, the departure times of all the jobs in the block decrease, i.e., $x_{i} \leq x_{i, L}$ for all $i=1, \ldots, L$.

\section{REFERENCES}

[1] R. Alur, T. A. Henzinger, and E. D. Sontag, Eds., Hybrid Systems. New York: Springer-Verlag, 1996

[2] P. Ansaklis, W. Kohn, M. Lemmon, A. Nerode, and S. Sastry, Eds., Hybrid Systems V. New York: Springer-Verlag, 1998.

[3] M. S. Branicky, V. S. Borkar, and S. K. Mitter, "A unified framework for hybrid control: Model and optimal control theory," IEEE Trans. Automat. Contr., vol. 43, pp. 31-45, Jan. 1998.

[4] A. E. Bryson and Y. C. Ho, Applied Optimal Control: Optimization, Estimation, and Control. Bristol, PA: Hemisphere, 1975.

[5] C. G. Cassandras, Discrete Event Systems: Modeling and Performance Analysis. Homewood, IL: Irwin, 1993. 
[6] F. H. Clarke, Optimization and Nonsmooth Analysis. New York: Wiley-Interscience, 1983.

[7] Y. Cho and C. G. Cassandras, "Optimal control for steel annealing processes as hybrid systems," in 39th IEEE Conf. Decision Control, 2000.

[8] Y. Cho, C. G. Cassandras, and D. L. Pepyne, "Forward decomposition algorithms for optimal control of a class of hybrid systems," Int. J. Robust Nonlin. Control, vol. 2, pp. 369-394, 2001.

[9] C. D. Kelly, D. Watanapongse, and K. M. Gaskey, "Application of modern control to a continuous anneal line," IEEE Control Syst. Mag., vol. 8, pp. 32-37, 1988 .

[10] M. Gazarik and Y. Wardi, "Optimal release times in a single server: An optimal control perspective," IEEE Trans. Automat. Contr., vol. 43, pp. 998-1002, July 1998.

[11] K. Gokbayrak and C. G. Cassandras, "Hybrid controllers for hierarchically decomposed systems," in Proc. 3rd Int. Workshop Hybrid Systems. Computation Control, 2000, pp. 117-129.

[12] R. L. Grossman, A. Nerode, A. P. Ravn, and H. Rischel, Eds., Hybrid Systems. New York: Springer-Verlag, 1993.

[13] D. E. Kirk, Optimal Control Theory. Englewood Cliffs, NJ: PrenticeHall, 1970.

[14] L. Kleinrock, Queueing Systems, Volume I: Theory. New York: WileyInterscience, 1975.

[15] M. M. Makela and P. Neittaanmaki, Nonsmooth Optimization. Singapore: World Scientific, 1992.

[16] D. L. Pepyne, "Performance Optimization Strategies for Discrete Event and Hybrid Systems," Ph.D. dissertation, Dept. of Electrical and Computer Engineering, University of Massachusetts, Amherst, MA, Feb. 1999.

[17] D. L. Pepyne and C. G. Cassandras, "Modeling, analysis, and optimal control of a class of hybrid systems," J. Discrete Event Dyna. Syst., vol. 8, pp. 175-201, 1998.

[18] — , "Hybrid systems in manufacturing," Proc. IEEE, vol. 88, pp. 1108-1123, July 200.

[19] R. T. Rockafellar, "Convex analysis," in Princeton Mathematics Series. Princeton, NJ: Princeton University Press, 1970, vol. 28.

[20] Y. Wardi, D. L. Pepyne, and C. G. Cassandras, "A backward algorithm for computing optimal controls for single-stage manufacturing systems,” Int. J. Prod. Res., 2001, to be published.

[21] N. Yoshitani, "Model-based control of strip temperature for the heating furnace in continuous annealing," IEEE Trans. Contr. Syst. Technol., vol. 6, pp. 146-156, Mar. 1998.

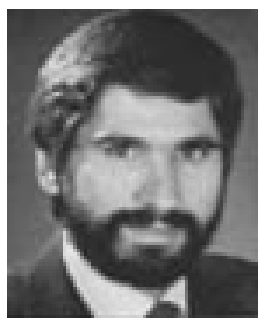

Christos G. Cassandras (S'82-M'82-SM'91-F'96) received the B.S. degree from Yale University, New Haven, CT, the M.S.E.E. degree from Stanford University, Stanford, CA, and the S.M. and Ph.D. degrees from Harvard University, Cambridge, MA, in 1977, 1978, 1978, and 1982, respectively.

From 1982 to 1984, he was with ITP Boston, Inc. where he worked on the design of automated manufacturing systems. From 1984 to 1996, he was a Faculty Member with the Department of Electrical and Computer Engineering, University of Massachusetts, Amherst. Currently, he is Professor of manufacturing engineering and Professor of electrical and computer engineering at Boston University, Boston, MA. He specializes in discrete event systems, stochastic optimization, and computer simulation, with applications to computer networks, manufacturing systems, and transportation systems. He has published over 150 papers in these areas, and two textbooks, one of which was awarded the 1999 Harold Chestnut Prize by the IFAC.

Dr. Cassandras is currently Editor-in-Chief of the IEEE TRANSACTIONS ON AUTOMATIC CONTROL and has served on several editorial boards and as Guest Editor for various journals. He is a member of the CSS Board of Governors. He was awarded a 1991 Lilly Fellowship, and is also a member of Phi Beta Kappa and Tau Beta Pi.

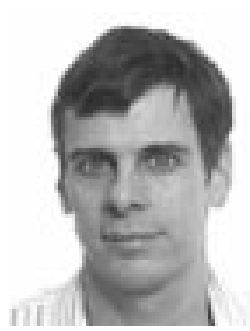

David L. Pepyne (S'91-M'92) received the B.S. degree from the University of Hartford, CT, in 1986 and the M.S. and Ph.D. degrees from the University of Massachusetts, Amherst in 1995 and 1999, respectively, all in engineering.

From 1986 to 1990, he was an Officer with the U.S. Air Force, stationed at Edwards A.F.B., CA, and working as a Flight Test Engineer. From 1995 to 1997, he was a Project Engineer with Alphatech, Inc., Burlington, MA. Currently, he is a Research Fellow in the Division of Engineering and Applied Science at Harvard University, Cambridge, MA, where his research focuses on complex systems, intrusion and fault detection, optimization theory, and optimal control of discrete event and hybrid systems.

Dr. Pepyne is currently an Associate Editor for the IEEE Control Systems Society Conference Editorial Board.

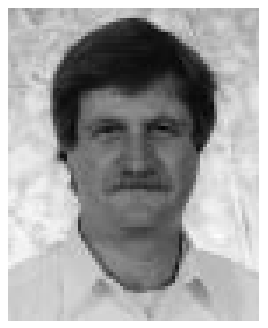

Yorai Wardi received the Ph.D. degree in electrical engineering and computer sciences form the University of California, Berkeley, in 1982.

From 1982 to 1984 , he was a Member of the Technical Staff at Bell Telephone Laboratories and Bell Communications Research, Holmdel, NJ. Since 1984, he has been with the School of Electrical and Computer Engineering at the Georgia Institute of Technology, Atlanta, where he is currently an Associate Professor. He spent the 1987-1988 academic year at the Department of Industrial Engineering and Management, Ben Gurion University of the Negev, Israel. His interests are in discrete event dynamic systems, perturbation analysis, and modeling and optimization of hybrid dynamical systems. 